\title{
The GOODS-MUSIC sample: a multicolour catalog of near-IR selected galaxies in the GOODS-South field $\star, \star \star$
}

\author{
A. Grazian ${ }^{1}$, A. Fontana ${ }^{1}$, C. De Santis ${ }^{1}$, M. Nonino ${ }^{2}$, S. Salimbeni ${ }^{1}$, E. Giallongo ${ }^{1}$, S. Cristiani ${ }^{2}$, \\ S. Gallozzi ${ }^{1}$, and E. Vanzella ${ }^{2}$
}

1 INAF - Osservatorio Astronomico di Roma, via Frascati 33, 00040 Monteporzio, Italy

e-mail: fontana@mporzio.astro.it

2 INAF - Osservatorio Astronomico di Trieste, via G.B. Tiepolo 11, 34131 Trieste, Italy

Received 3 August 2005 / Accepted 17 November 2005

\begin{abstract}
Aims. We present a high quality multiwavelength (from 0.3 to $8.0 \mu \mathrm{m}$ ) catalog of the large and deep area in the GOODS Southern Field covered by the deep near-IR observations obtained with the ESO VLT.

Methods. The catalog is entirely based on public data: in our analysis, we have included the $F 435 W, F 606 W, F 775 W$ and $F 850 L P$ ACS images, the $J H K$ s VLT data, the Spitzer data provided by IRAC instrument (3.6, 4.5, 5.8 and $8.0 \mu \mathrm{m})$, and publicly available $U$-band data from the 2.2ESO and VLT-VIMOS. We describe in detail the procedures adopted to obtain this multiwavelength catalog. In particular, we developed a specific software for the accurate "PSF-matching" of space and ground-based images of different resolution and depth (ConvPhot), of which we analyse performances and limitations. We have included both $z$-selected, as well as $K$ s-selected objects, yielding a unique, self-consistent catalog. The largest fraction of the sample is $90 \%$ complete at $z \simeq 26$ or $K \mathrm{~s} \simeq 23.8$ (AB scale). Finally, we cross-correlated our data with all the spectroscopic catalogs available to date, assigning a spectroscopic redshift to more than 1000 sources.

Results. The final catalog is made up of 14847 objects, at least 72 of which are known stars, 68 are AGNs, and 928 galaxies with spectroscopic redshift (668 galaxies with reliable redshift determination). We applied our photometric redshift code to this data set, and the comparison with the spectroscopic sample shows that the quality of the resulting photometric redshifts is excellent, with an average scatter of only 0.06 . The full catalog, which we named GOODS-MUSIC (MUltiwavelength Southern Infrared Catalog), including the spectroscopic information, is made publicly available, together with the software specifically designed to this end.
\end{abstract}

Key words. galaxies: distances and redshifts - galaxies: evolution - galaxies: high-redshift - galaxies: photometry - methods: data analysis techniques: image processing

\section{Introduction}

The advent of the Hubble Space Telescope and of the modern giant telescopes has opened a new era in observational cosmology, where galaxy evolution can be traced back to its early stages. In this context, deep multicolour imaging surveys are established as a powerful tool to access the population of faint galaxies with relatively high efficiency. An already classical technique (Steidel et al. 1995; Madau et al. 1998) is used to select high redshift galaxy candidates with sharp colour selection criteria, which has been shown to be particularly efficient at $z \simeq 3-4$ (Steidel et al. 1999).

Other strategies have focused on surveys that sample the whole spectral range from the $U$ to the $K$ band, enabling galaxy

\footnotetext{
* Full Tables 5-7 are available in electronic form at the CDS via anonymous ftp to cdsarc.u-strasbg.fr $(130.79 .128 .5)$ or via http://cdsweb.u-strasbg.fr/cgi-bin/qcat?J/A+A/449/951

$\star \star$ Appendix is only available in electronic form at http://www.edpsciences.org
}

evolution to be followed on a wider range of redshifts, and that have triggered the development and use of photometric redshifts to extract information about galaxies at any redshift. The Hubble Deep Field North (HDFN), and the subsequent Hubble Deep Field South (HDFS), are the most successful examples of this approach, which has stimulated an impressively large number of scientific investigations (see Ferguson et al. 2000 for a review).

The Great Observatories Origins Deep Survey (GOODS, Dickinson 2001) represents the next generation of this kind of survey. It is based on observations of two separated fields of $10 \times 15$ arcmin each, centred on the HDFN and Chandra Deep Field South (CDFS hereafter), respectively. Over a total area that is about 50 times the combined HDFN/S, it is the result of an impressive effort with the most advanced facilities.

The available dataset includes ultradeep images from ACS on HST, from the mid-IR satellite Spitzer, from the ultraviolet satellite GALEX, from the X-ray satellites Chandra and XMM, as well as from a long number of ground-based 
facilities (see Giavalisco et al. 2004 for a more detailed presentation). Spectroscopic follow-up is also available or ongoing from a list of surveys and facilities (Cimatti et al. 2002; Cowie et al. 2004; Vanzella et al. 2005).

In particular, the GOODS southern pointing, located over the CDFS (centred on $\alpha=03: 32: 30 \delta=-27: 48: 30$, Giacconi et al. 2000) and encompassing the ACS Ultra Deep Field (UDF hereafter, Beckwith et al. 2003) and the GMASS ${ }^{1}$ ESO Large Program (Galaxy Mass Assembly ultradeep Spectroscopic Survey, PI Cimatti), has been the target of extensive observations with ESO telescopes, carried on in the spirit of public surveys. To date, a major effort has been devoted to deep imaging observations in the $J, H$, and $K$ s bands with the VLT-ISAAC infrared imager. The final area covered by the $K$ s images approaches $140 \mathrm{arcmin}^{2}$ to a $1 \sigma$ limiting depth of typically $26-27 \mathrm{mag} / \operatorname{arcsec}^{2}$ (AB scale), making it a unique combination of depth and size in the near-IR. Additional observations in the $U$ band have also been obtained, and a large spectroscopic follow-up is currently underway.

This paper describes the multicolour catalog that we obtained using the public imaging data available in the GOODS-CDFS region. It includes two $U$ images obtained with the ESO $2.2 \mathrm{~m}$ telescope and one $U$ band image from VLT-VIMOS, the ACS-HST images in four bands, the VLT-ISAAC $J, H$, and $K \mathrm{~s}$ bands as well as the Spitzer images in at 3.5, 4.5, 5.8, and $8 \mu \mathrm{m}$. Most of these images have been made publicly available in the coadded version by the GOODS team, while the $U$ band data were retrieved in raw format and reduced by our team.

From this dataset we obtained both a $z$-selected, as well as a $K$ s-selected, sample. We also collected all the available spectroscopic information and cross-correlated the spectroscopic redshifts with our photometric catalog. For the unobserved fraction of the objects, we applied our photometric redshifts code to obtain well-calibrated photometric redshifts, which are described in a forthcoming paper (Vanzella et al. in preparation), together with the redshift based on the neural network technique (Vanzella et al. 2004).

The resulting dataset will be used for several types of scientific output, for which we refer to forthcoming papers. At the same time, we make the dataset publicly available, along with some software specifically developed for this project, all of which can be of wider interest, and which we first describe here.

The paper is organised as follows. In Sect. 2, we briefly summarise the available dataset. In Sect. 3, we discuss the procedure for object detection in the ACS- $z$ image, and the corresponding measurement of total magnitude. In Sect. 4 , we discuss the procedure adopted to estimate colours of $z$-selected objects, which has requiered the development of a new software code, which we explain in some detail. In Sect. 5, we focus on the $K$ s-selected sample, describing how we identify the objects that are detected in $K \mathrm{~s}$ and not in $z$, and how we measure their total magnitude and colours. In Sect. 6 we briefly list the spectroscopic observations that we used to assign

\footnotetext{
${ }^{1}$ http://www.arcetri.astro.it/ cimatti/gmass/ gmass.html
}

spectroscopic redshifts, while Sect. 7 discusses the photometric redshift technique applied. Section 8 finally describes the format and the technical details adopted for the public release.

All magnitudes are given in $\mathrm{AB}$ scale, and a $\Lambda$-dominated cosmology $\left(\Omega_{\mathrm{m}}=0.3, \Omega_{\Lambda}=0.7\right.$ and $\left.H_{0}=70 \mathrm{~km} \mathrm{~s}^{-1} \mathrm{Mpc}^{-1}\right)$ has been adopted throughout the paper.

The data and photometric redshift cata$\operatorname{logs}$ are also available on line at the WEB site http://lbc.oa-roma.inaf.it/mwcwi.

\section{Imaging data}

\subsection{GOODS-south ACS data}

In this paper we will focus on the southern field of the GOODS survey, located in the CDFS.

Images are made available for all 4 GOODS filters, which are those used in the UDF observations: $F 435 W, F 606 W$, $F 775 W$, and $F 850 L P$. In the following, we will refer to these images as $B, V, i$, and $z$, respectively. Of this data set, in this work we use the data release 1.0 provided by the GOODS team (Giavalisco et al. 2004). Table 1 summarises the characteristic of the each pointing of the GOODS ACS data.

The V1.0 ACS GOODS images released by the GOODS team were divided into a grid of 4 by $58 \mathrm{~K} \times 8 \mathrm{~K}$ frames for each band. Since we were only interested in the portion of the image covered by the $J$ and $K$ s observations, we considered only the tiles that had an overlap (at least partial) with the IR images. Of these images, we built a single mosaic for each band (the software used for this purpose, named fitstile, is available on our web site): each resulting frame is $3.4 \mathrm{~Gb}$ wide, which can still be handled by SExtractor in a single run, provided it is compiled with the "large file" option. Although this solution is somewhat impractical (for instance, common visualisation tools fail to display such large images), it allows us to avoid the complications arising when catalogs are obtained on overlapping images, such as inhomogeneities in the large scale background, different object detection, and other border effects.

\subsection{The ISAAC data}

The GOODS field of the CDFS was the target of a deep imaging campaign in the near infrared with the ESO telescopes. A large field (20 by 20 arcmin) was covered with SOFI at a shallow magnitude limits in the $J, H$, and $K$ s bands. The GOODS-CDFS is being covered by much deeper observations in the same NIR bands with the ISAAC instrument. These data have been partially released by ESO and will be used in this work. A full documentation of this data set is presented in Vandame et al. (in preparation), while details of the on-going GOODS program at ESO are given at http: //www . eso.org/science/goods/products.html.

The version 1.0 data release, analysed with the ESOMVM pipeline for imaging data reduction (Vandame 2002), includes 22 fully reduced VLT/ISAAC fields in $J$ and $K$ s bands covering about $140 \operatorname{arcmin}^{2}$ of the GOODS region in the CDFS. 
Table 1. A summary of the photometric data of the GOODS-South field used in this work.

\begin{tabular}{lcccccccc}
\hline \hline FILTER & $\begin{array}{c}\lambda_{\mathrm{c}} \\
\AA\end{array}$ & $\begin{array}{c}\Delta \lambda \\
\AA\end{array}$ & $\begin{array}{c}\text { EXPTIME } \\
\mathrm{A}\end{array}$ & $\begin{array}{c}F W H M^{a} \\
\operatorname{sicsec}\end{array}$ & $\begin{array}{c}\text { PIXSCALE } \\
\operatorname{arcsec} / \mathrm{px}\end{array}$ & $\begin{array}{c}\text { ZP } \\
\text { AB }\end{array}$ & $\begin{array}{c}\text { AREA }^{2} \\
\operatorname{arcmin}^{2}\end{array}$ & $\begin{array}{c}\text { MAGLIM }^{b} \\
90 \%\end{array}$ \\
\hline$U_{35}$ & 3590 & 222 & 53654 & 0.90 & 0.23 & 28.520 & 143.2 & 25.5 \\
$U_{38}$ & 3680 & 170 & 75100 & 1.10 & 0.23 & 28.755 & 143.2 & 24.5 \\
$U_{V I M O S}$ & 3780 & 197 & 10000 & 0.80 & 0.20 & 32.500 & 90.2 & 26.5 \\
$B(F 435 W)$ & 4330 & 508 & 7200 & 0.12 & 0.03 & 25.65288 & 143.2 & 27.5 \\
$V(F 606 W)$ & 5940 & 1168 & 6000 & 0.12 & 0.03 & 26.49341 & 143.2 & 27.5 \\
$i(F 775 W)$ & 7710 & 710 & 6000 & 0.12 & 0.03 & 25.64053 & 143.2 & 26.5 \\
$z(F 850 L P)$ & 8860 & 554 & 12000 & 0.12 & 0.03 & 24.84315 & 143.2 & 26.0 \\
$J_{\text {ISAAC }}$ & 12550 & 1499 & $12600^{c}$ & $0.45^{c}$ & 0.15 & 26.000 & 143.2 & 24.5 \\
$H_{\text {ISAAC }}$ & 16560 & 1479 & $18000^{c}$ & $0.45^{c}$ & 0.15 & 26.000 & 78.0 & 24.3 \\
$K s_{\text {ISAAC }}$ & 21630 & 1383 & $23400^{c}$ & $0.45^{c}$ & 0.15 & 26.000 & 143.2 & 23.8 \\
$C H 1_{\text {IRAC }}$ & 35620 & 3797 & 82800 & 1.60 & 0.60 & 22.416 & 143.2 & 24.0 \\
$C H 2_{\text {IRAC }}$ & 45120 & 5043 & 82800 & 1.70 & 0.60 & 22.195 & 143.2 & 23.4 \\
$C H 3_{\text {IRAC }}$ & 56860 & 6846 & 82800 & 1.90 & 0.60 & 20.603 & 143.2 \\
$C H 4_{\text {IRAC }}$ & 79360 & 14797 & 82800 & 2.00 & 0.60 & 21.781 & 143.2 & 22.0 \\
\hline
\end{tabular}

${ }^{a}$ For ground-based images, $F W H M$ corresponds to the seeing, while for space-based data it is the PSF of the instrument.

${ }^{b}$ The limiting magnitudes (at 90\% completeness) are the mean values on the field, averaged on the positions and areas of every object.

${ }^{c}$ For $J, H$, and $K$ s filters, we report only the mean value of seeing and exposure time: detailed values can be found in Vandame et al. (in preparation).

Figure 1 shows the position of the released ISAAC fields (yellow) superimposed on the optical image of the CDFS by ACS. The cyan square shows the position of the UDF on the GOODS-CDFS field, while the red rectangle shows the layout of the K20 survey. The four white quadrants show the actual coverage of the VIMOS $U$ band imaging. The $H$ band observations cover only half of the entire survey, and are only limited to twelve fields. They have not been released by the present V1.0 data release but were part of a previous (V0.5) EIS data release and are public on the ESO archive.

The typical exposure times range from 3 to $6 \mathrm{~h}$, the seeing ranges from 0.4 to 0.6 arcsec, and it is typically below 0.5 arcsec. We measured the value of the seeing for each ISAAC field by averaging all the bright stellar objects from the image, after normalisation of their profiles to unit flux. For each ISAAC field, we also obtained a "convolution kernel" $\mathcal{K}$, which is the transformation matrix that converts the ACS PSF to the relevant ISAAC one that we shall use for the colour estimate. This transformation was computed in Fourier space, as $\mathcal{K}^{\prime}=P S F_{\mathrm{ISAAC}}^{\prime} / P S F_{\mathrm{ACS}}^{\prime}$, where the prime refers to the Fourier transform. An optimal Wiener filter, which suppresses the high frequency fluctuations, was applied in the Fourier domain to remove the effects of noise.

\subsection{U-band data: 2.2-WFI and VLT-VIMOS}

We searched the ESO public archives for deep imaging with the aim of complementing the spectral coverage by the GOODS team. Particularly important is the $U$ band, which improves the photometric redshift estimate, in particular for the lowest redshift $(z \leq 0.5)$, when the $U$ band still probes the blue side of the
$4000 \AA$ Å break and, most important, is fundamental for identifying $z \sim 3$ galaxies ( $U$-dropouts).

In the ESO Science Archive there are $U$-band images taken with the wide field imager (WFI) at La Silla (Chile), which are part of the EIS public survey (Arnouts et al. 2001), as well as recent images with the VLT-VIMOS imager.

The WFI images were obtained in two filters, the socalled $U_{35}$ and $U_{38}$. The $U_{38}$ is the standard Bessel $\mathrm{U}$ with a peak efficiency of $50 \%$, centred around $\lambda_{\mathrm{c}}=3800 \AA$, while the $U_{35}$ is a bit bluer, $\lambda_{c}=3500 \AA$, with higher efficiency $(\sim 80 \%)$, but with a red leak at $\lambda \geq 8000 \AA$, where the WFI CCD is still sensitive. The net exposure time is $53654 \mathrm{~s}$ $(\sim 15 \mathrm{~h})$ and $75100 \mathrm{~s}(\sim 21 \mathrm{~h})$ for the $U_{35}$ and $U_{38}$ filters, respectively. The $U$ band image of VIMOS is based on a redder filter $\left(\lambda_{\mathrm{c}}=3900 \AA\right)$ and has an exposure time of $10000 \mathrm{~s}$ in total. The coverage of the GOODS-CDFS field, however, is partial, since the observing program has not yet been completed.

We decided to use these images $\left(U_{35}\right)$ even if they are affected by red leakage: by inserting the correct (with red leak) filter transmission curve to the photometric redshift procedure one is able to mimic the behaviour of distant galaxies in the $U_{35}$ filter. The VIMOS images available on Dec. 2004 cover a significant fraction of the field (about 60\%), although they exhibit the large gaps arising from the array disposition (see Fig. 1).

The raw $U$-band images were collected from the ESO Science Archive and reduced using Python scripts and the IRAF task mscred following the instruction of Valdes (2002). The total exposure times of these images surpass the previous data release of EIS-DPS, which only reached 43200 and $61200 \mathrm{~s}$ in the $U_{35}$ and $U_{38}$, respectively. The image quality 


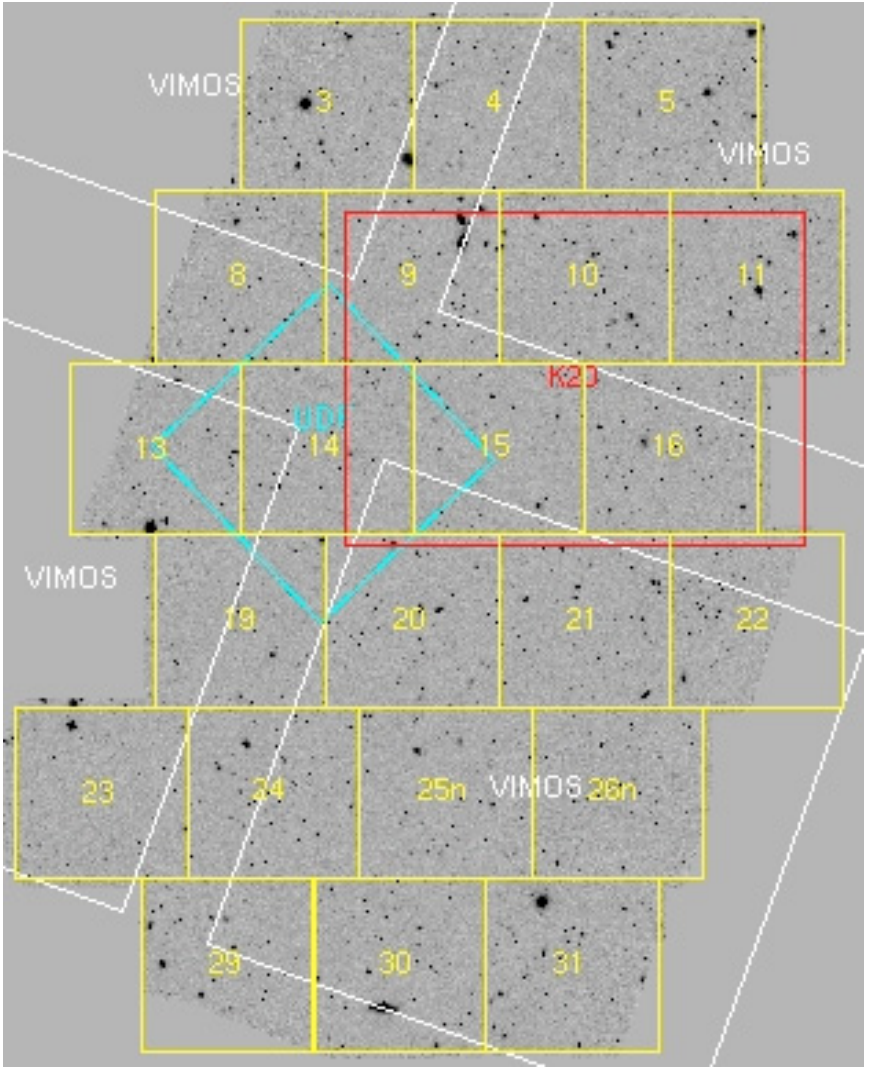

Fig. 1. The ACS GOODS-S field with superimposed the ISAAC tiling made by ESO to cover the whole field in $J$ and $K$ s. The field shown is the ACS in the $z$-band limited to the area common to ACS and ISAAC observations. The cyan square marks the position of the ACS UDF. The NICMOS UDF Treasury observations cover a field that is inside the UDF. The red rectangle marks the position of the K20 survey. The four white quadrants show the actual coverage of VLT-VIMOS $U$ band imaging; large gaps are visible, since the observing program has not yet been completed.

of these images is good, with a seeing of 0.9-1.1 arcsec and a magnitude limit of 26.5-27.0 (AB mag) at 5 sigma in a photometric aperture of 3 arcsec. The $U$ image of VIMOS had good seeing conditions $(0.7-0.8$ arcsec $)$ and reached a deeper magnitude limit (28 AB, $5 \sigma)$.

\subsection{IRAC data from the Spitzer Legacy Program}

The GOODS survey also incorporates a Spitzer Space Telescope Legacy Program to carry out the deepest observations with this facility at 3.6 to 24 microns, to study galaxy formation and evolution over a wide range of redshift and cosmic lookback time.

The first and second Spitzer data releases (DR1 and DR2) consist of "best-effort" reductions of data taken with the infrared array camera (IRAC, Fazio et al. 2004) on-board Spitzer. These are images from the two epochs of the "superdeep" IRAC observations for each of the two GOODS fields (Dickinson et al., in preparation).

These fields were imaged at $3.6-8 \mu$ with IRAC, with a mean exposure time per position of approximately $23 \mathrm{~h}$ per

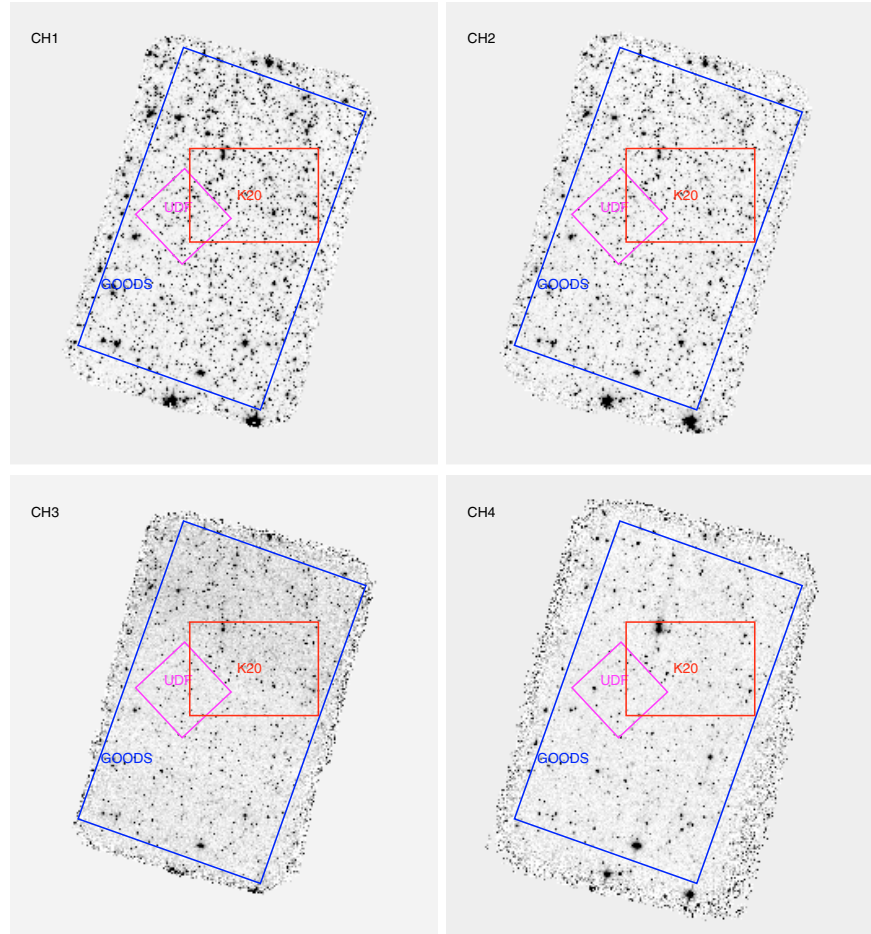

Fig. 2. The four channels of the IRAC images for the GOODS-South field. The pointings are chosen to have the UDF in the overlapping regions, where the exposure time is twice that in the external parts. The K20 layout is shown, as well.

band (doubled in the overlap strip containing the UDF), reaching far deeper flux limits than observations planned for the Guaranteed Time programs.

The IRAC observes simultaneously in all four channels, with channels 1 and 3 (3.6 and 5.8 microns) covering one pointing on the sky, and channels 2 and 4 (4.5 and 8.0 microns) covering another. The two IRAC fields of view are separated by about 6.7 arcmin in the focal plane, and the long axes of the GOODS fields are oriented along the direction separating the two IRAC fields of view. The consequence of the $2 \times 2$ mapping pattern is that, in a given observing epoch, the area covered by channels $1+3$ and the one covered by channels $2+4$ have a small region of overlap (about $3 \mathrm{arcmin}$ ). The GOODS-S four-channel overlap region includes the Hubble UDF (Beckwith et al. 2003).

The first and second epochs of IRAC images publicly released by the GOODS Team, cover the entire GOODS-CDFS field at 3.6, 4.5, 5.8 and $8.0 \mu \mathrm{m}$ (see Fig. 2). Table 1 gives detailed information for the GOODS-CDFS survey used in this paper, with the wavelengths, area covered, and magnitude limits for all the filters available at this moment.

\subsection{Accurate estimation of photometric errors}

The correct evaluation of the photometric errors for astronomical sources is bound to the possibility of having a realistic variance image associated with the measure image. It has become a common feature of the data reduction pipelines to produce "weight map" images that are usually proportional to the 
exposure time of each pixel and, therefore, provide a measure of the relative $\mathrm{S} / \mathrm{N}$ across the image. These images can be used by SExtractor to obtain a first map of the background rms, which is the main source of noise for faint sources. However, the interpolations introduced by drizzling the images (shifting, rotating, correcting distortion, and subsampling pixels onto a finer grid) result in correlations between pixels in the drizzled science images. Therefore, the apparent rms background noise that one measures in the image is smaller than the actual rms, due to the effects of these correlations (for a more detailed discussion of weight map conventions and noise correlation in drizzling, please see Casertano et al. 2000, especially Sect. 3.5 and Appendix A).

To overcome this problem, we used a simple and efficient method to derive the rms from the scientific image itself, based on the correlation matrix of the pixels of the background. Indeed, as we explain better in Appendix A, the true rms of an image is given by:

$\sigma_{\text {true }}^{2}=\sum_{j=-m}^{+m} \frac{1}{N} \sum_{i=1}^{N} y_{i} y_{i+j}$,

where $N$ is the number of pixels used for the rms calculation, $y_{i}$ the value of the $i$ th pixel on the source-subtracted image, and $m$ the correlation length of the noise, typically of 10-20 pixels.

In practice, we used SExtractor to convert the weight maps (available for each image of our data set) into the corresponding image of apparent rms. We then estimated the correlation matrix in a homogeneous section of the image to compute the accurate rms, and finally normalised the apparent rms image accordingly.

We verified this procedure in the ACS images, where the weight images (Giavalisco et al. 2004) coincide with the expected inverse variance maps (i.e., $1 / \sigma^{2}$ ) per pixel. In this case, we obtained an absolute rms of 0.001396 , comparable to the value 0.0014 obtained with a traditional approach ${ }^{2}$, as well as with the publicly available weight maps (i.e., $1 / \sigma^{2}$ ).

For the U, ISAAC, and Spitzer images, we applied the same technique and found that the true rms is typically a factor 1.4 times higher than the rms that was directly measured on the science frames. As explained above, in the case of the ACS images, we used the available weight maps to compute the rms image by simply inverting the square root of the weight map.

\subsection{Magnitude limits and effective areas}

The total area of the survey is the intersection of the images in $B, V, i, z, J$, and $K$ s bands. The $U_{35}$ and $U_{38}$ images coves a much larger field of view (FoV), approximately 30 by 30 arcmin, the IRAC images completely cover the field layout, while the VIMOS- $U$ and the $H$ band cover about half of the total field. Images in $V, i$, and $z$ cover the same area, while $B$ is smaller due to the lower number of dithering steps. Since we

\footnotetext{
${ }^{2}$ We polluted the $z$ band images of GOODS with synthetic stars of different magnitudes and derived the true magnitude errors by the variance over 100 realisations.
}

are interested in $z$ and $K$ s-selected samples with good photometric redshift accuracy, we limit the sample to the area given by the intersection of the $B$ and $K$ s images. In this sample, all the objects have $U_{35} U_{38} B V i z J K s$ and IRAC coverage, and about a half also have VIMOS- $U$ and $H$ band observations. The total area is $143.2 \mathrm{sq}$. arcmin. and the layout of the resulting field is shown in Fig. 1.

Associating a limiting magnitude to this area is not trivial, since the exposure maps in optical and NIR are different and very complicated. We first decided to estimate an accurate map of the magnitude limits as a function of position using rms images in $z$ and $K \mathrm{~s}$. The limiting magnitudes are computed at 1 sigma level and in an area of 1 sq. arcsec. Figures 3 and 4 show this in the GOODS area, as well as the relevant distribution of the magnitude limit. Dark areas represent deeper exposures that correspond to the faintest magnitude limits. In the $z$ band image we identified 6 main areas with a different interval in the magnitude limits; in $K$ s band the magnitude limit intervals identified are 6 (see Table 2).

This information was used as follows. We associated the $1-\sigma$ magnitude limit to each detected object (either in the $z$ or in the $K$ s-selected sample) as obtained from the "magnitude limit maps" at the corresponding position. The simulations described in Sect. 3 made it possible to translate this $1-\sigma$ magnitude limit into a completeness limit for the object detection. Each catalog was therefore separated according to the magnitude limit, using the bins listed in Table 2. As a result, each catalog is actually the combination of 6 (for $z$ and for $K \mathrm{~s}$ ) catalogs with different magnitude limits. They should be used as independent catalogs with different magnitude limits and areas. This solution can be easily applied to all the cases where volume-sensitive statistics have to be applied, as in the case of luminosity densities or luminosity functions. Analyses that are sensitive to the position information (as clustering) instead require the use of the "magnitude limit maps". In the publicly released catalog we provide the corresponding magnitude limit both in $z$ as in $K$ s with each object.

\section{Object detection}

\subsection{ACS detection}

The $z$ band of ACS GOODS frames was used as a detection image to build a catalog for the GOODS-South field. The advantages are twofold: first, the morphological details and the resolution of the space-based images cannot be obtained with currently available ground-based instruments in the optical. Second, the longest wavelength available in the ACS instrument makes it possible to detect high redshift objects ( $i$-dropout). Although fainter sources in the $i$ band exist that are not or barely detected in the $z$ band images, the significance of their detection is less than $90 \%$ complete, so they cannot be used as a statistically complete sample and are not considered here.

The optical catalog of GOODS-South field has been produced running SExtractor (Bertin \& Arnouts 1996) using the $z$ band big mosaic as detection image. We used an external flag image to eliminate the borders of the GOODS field. 

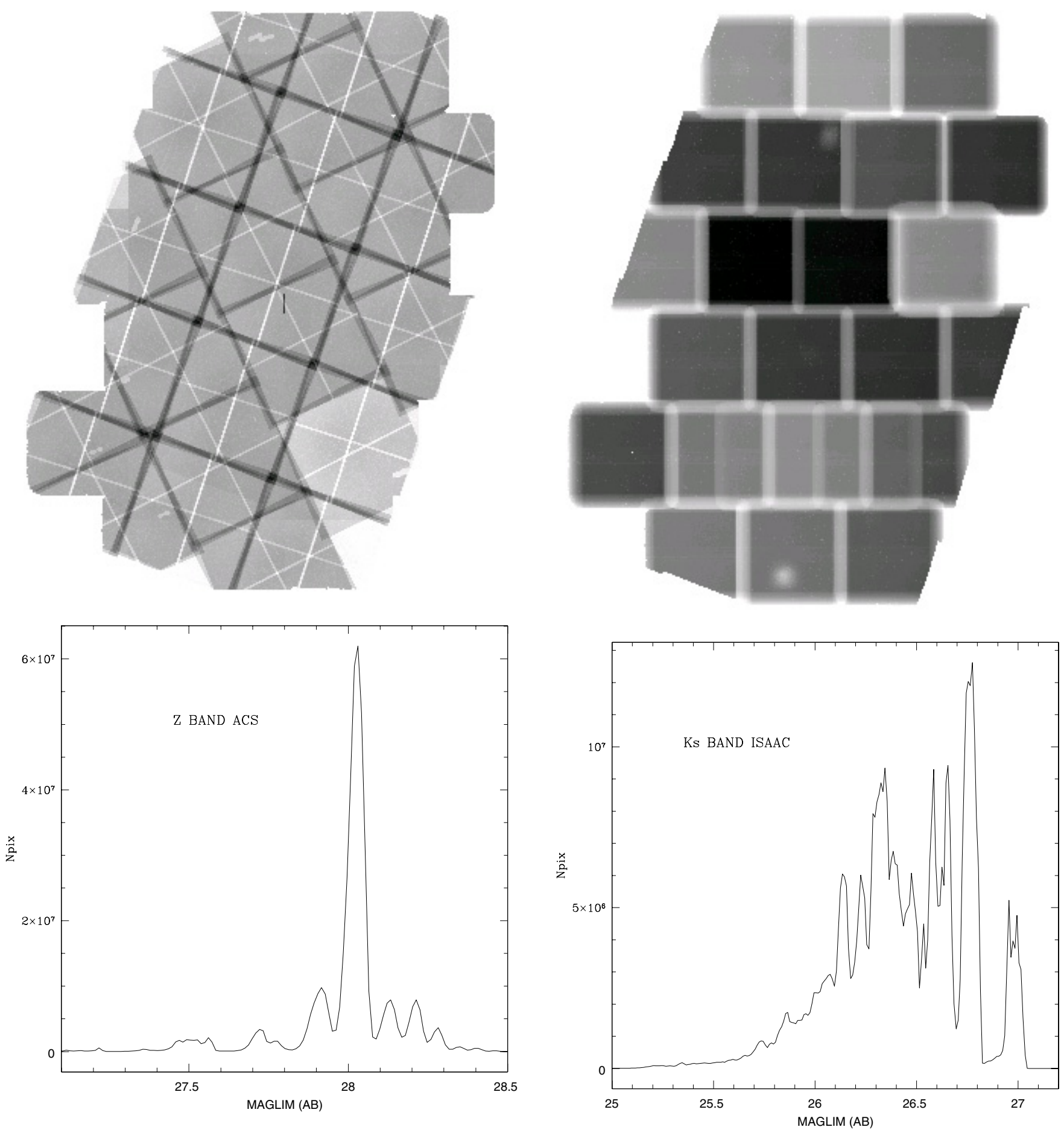

Fig. 3. The magnitude limits (at 1 sigma and in 1 sq. arcsec) for the $z$ band in the GOODS area. Dark areas correspond to deeper exposures, while white zones are much shallower ones, as also shown in the histogram of the magnitude limits. The geometry of the dark and white areas depends on the dithering strategy adopted.

Because of the large number of tunable parameters, the use of SExtractor on a given data set is not straightforward. After the first checks on the catalog, we realized that it is impossible to find a unique set of parameters to obtain an optimal detection over large FoVs, since in large and deep areas the objects have a wide range of dimensions, going from the small and faint objects to the large, low-surface brightness but with

Fig. 4. The magnitude limits (at 1 sigma and in 1 sq. arcsec) for the $K$ s band in the GOODS area. Dark areas correspond to deeper exposures, while white zones are much shallower ones, as also shown in the histogram of the magnitude limits.

high total luminosity near galaxies. In particular, the most critical parameters are those regulating the deblending and extension of the object (DETECT-MINAREA, DETECT-THRESH, DEBLEND-MINCONT, and DEBLEND-NTHRESH).

To provide an optimal detection technique throughout all the GOODS field, we modified the SExtractor code, following our experience on the HDFS, described by 
Table 2. Magnitude intervals that we used to divide the overall sample into subsamples with well-defined magnitude limits and corresponding areas for the $z$ and $K$ s bands. See also Figs. 3 and 4. MAGLIMI and MAGLIM2 (in magnitudes per sq. arcsec) define the limits of each magnitude bin. MAGTOT is the corresponding limit for the total magnitude of the objects, obtained using the simulations described in Sect. 3.2.

\begin{tabular}{lcccc}
\hline \hline FILTER & $\begin{array}{c}\text { MAGLIM1 } \\
1 \sigma\end{array}$ & $\begin{array}{c}\text { MAGLIM2 } \\
1 \sigma\end{array}$ & $\begin{array}{c}\text { AREA } \\
\operatorname{arcmin}^{2}\end{array}$ & MAGTOT \\
\hline$z$ & 26.65 & 27.65 & 7.16667 & 24.65 \\
$z$ & 27.65 & 27.85 & 6.40742 & 25.65 \\
$z$ & 27.85 & 27.95 & 15.4276 & 25.85 \\
$z$ & 27.95 & 28.10 & 89.9600 & 25.95 \\
$z$ & 28.10 & 28.18 & 10.3641 & 26.10 \\
$z$ & 28.18 & 28.90 & 13.8741 & 26.18 \\
\hline$K \mathrm{~s}$ & 24.70 & 26.08 & 10.7698 & 21.60 \\
$K \mathrm{~s}$ & 26.08 & 26.20 & 8.95936 & 22.98 \\
$K \mathrm{~s}$ & 26.20 & 26.50 & 40.5733 & 23.10 \\
$K \mathrm{~s}$ & 26.50 & 26.70 & 38.2161 & 23.40 \\
$K \mathrm{~s}$ & 26.70 & 26.90 & 34.8572 & 23.60 \\
$K \mathrm{~s}$ & 26.90 & 27.10 & 9.82067 & 23.80 \\
\hline
\end{tabular}

Vanzella et al. (2001). The idea is to adopt a general set of SExtractor parameters for the global image and to define small areas (subframes) where a set of ad hoc parameters are adopted to optimise the detection of problematic objects. In this case we adopted a set of parameters tuned to detect the faint, compact objects to obtain the bulk of the catalog. With these parameters, SExtractor typically oversplits bright, extended, and irregular sources (such as face-on spirals or low-surface brightness objects) in many fragments. To detect these objects, we obtained another version of the catalog using an extreme set of parameters (i.e. requesting very large area and no deblending) and visually inspected all the objects with magnitude $z<23$ and all the objects with spectroscopic redshift. In about $15 \%$ of the inspected objects we decided to adopt tailored SExtractor parameters in a corresponding "subframe". An investigation at fainter magnitudes has shown that such cases become very rare at $z>23$, so we ignored them.

We also slightly modified the SExtractor code to ensure that the "back-annulus" is at least 1 arcsec wide (as suggested by the GOODS team) and to correct a bug for the estimation of the isophotal-corrected magnitude in the "dual image" mode when the rms of detection and measure image differ by several orders of magnitude (see also the SExtractor mailing list).

Turning again to the global SExtractor configurations, we adopted the parameters listed in Table 3. The adopted value for DETECT_THRES H corresponds to a $3.5 \sigma$ detection over an area equal to 13 pixels. The high value for the cleaning parameter is due to the need to have connected pixels in the SEGMENTATION image: with a lower cleaning parameter it happens that a segmentation with the same identification number can be disconnected (not contiguous pixels).

This parameter set was derived after detailed simulations and inspections of the background-subtracted and
Table 3. SExtractor parameters for detection in the $z$ band.

\begin{tabular}{cc}
\hline \hline PARAMETER & VALUE \\
\hline DETECT_MINAREA & 13 \\
DETECT_THRESH & 0.9707254 \\
ANALYSIS_THRESH & 0.9707254 \\
DEBLEND_NTHRESH & 32 \\
DEBLEND_MINCONT & 0.05 \\
BACK_SIZE & 120 \\
BACK_FILTERSIZE & 9 \\
BACKPHOTO_THICK & 100 \\
CLEAN_PARAM & 3 \\
\hline
\end{tabular}

object-subtracted images, in order to maximise the completeness and to minimise the number of spurious objects or artifacts during the detection process. We note in particular that wide boxes for the background estimations are required to prevent large bright sources to distort the background map.

We compared our catalog with version r1.1 of the ACS multi-band source catalog released by the GOODS Team (Giavalisco et al. 2004). Excluding objects that are fainter than the detection limit, there are few galaxies with a difference of $1 \mathrm{mag}$ or more between our $z$ band estimation and the one provided by Giavalisco et al. (2004). These are systematically blended objects where we used the sub-frames with adaptive parameters to optimise the detection. Considering only isolated objects, the comparison gives $\left\langle\left|z-z_{r 1.1}\right|\right\rangle=0.01$ with $\sigma=0.12$. This result is not surprising, since both catalogs use the AUTO magnitudes provided by SExtractor as an estimate of the total magnitude. We made no attempt to correct for the known biases of the AUTO (or "Kron") magnitudes.

\subsection{Simulating the completeness of the detection}

We used a typical value for the rms in the $z$ band of 0.0014 (corresponding to a limiting magnitude of $z=28.18 \mathrm{AB}$ at 1 sigma in $1 \mathrm{sq}$. arcsec) to simulate the completeness of our detection criterion as a function of the magnitude of the sources and their size (half light radius). Note that $z=28.18$ as a magnitude limit corresponds to the deepest exposures for the $z$ band; for the less exposed areas, the completeness magnitude scales down to brighter values.

Figure 5 shows the $90 \%$ completeness level resulting from such simulations in the $z$ band, for both elliptical and spiral galaxies of different half-light radii and bulge/disk ratios. Comparing these selection functions with the observed distribution of magnitude and size for real objects in the GOODS field, we derived the exact value of the total magnitude $z$ at which our catalog is complete. Table 2 provides the total magnitudes to build a complete catalog of sources in the GOODS area.

The same exercise can be done in the $K$ s band images, where there are 6 main areas, with a broader range of magnitude limits. A typical value of 0.06 was used as the rms value for the simulations in the $K$ s band, corresponding to a limiting magnitude of 27.0 in a $1 \mathrm{sq}$. arcsec area at 1 sigma. This 

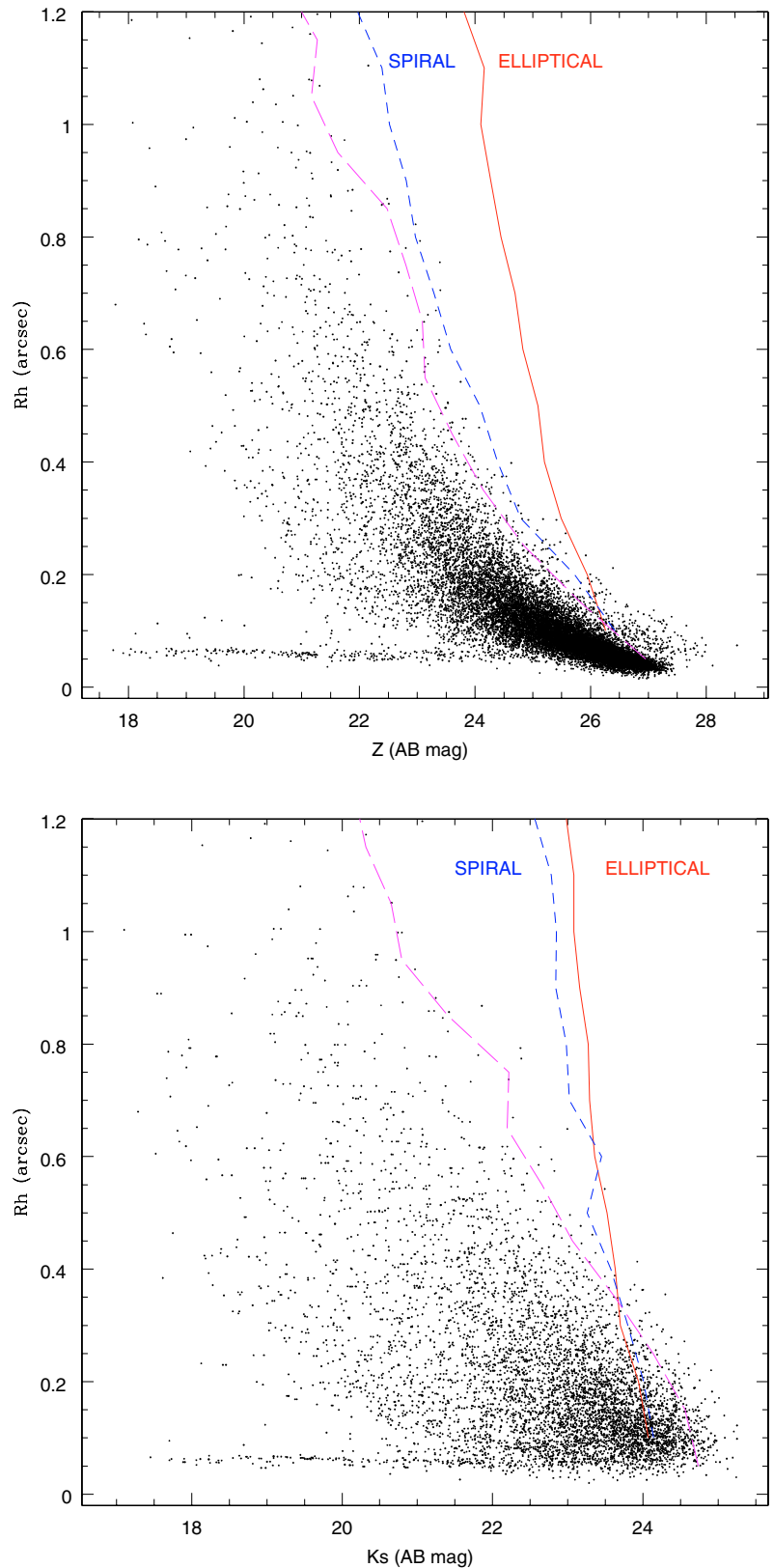

Fig. 5. The half-light radius of galaxies in the GOODS-CDFS region as a function of the observed $z$ and $K$ s magnitudes. The solid line indicates the completeness at $90 \%$ value for elliptical galaxies, while the short-dashed line shows the same for spiral galaxies. We simulated 100 galaxies for each bin of magnitude, half-light radius, and bulge/disk ratio: the magnitude range is from 20 to 30 in steps of 0.5 , the half-light radius range from 0.1 to 1.5 in steps of 0.1 arcsec, and the $\mathrm{B} / \mathrm{D}$ from 0.3 to 0.9 in steps of 0.1 for ellipticals or from 0.02 to 0.08 in steps of 0.01 for spiral galaxies. The completeness as a function of magnitudes and half-light radii is the fraction of galaxies recovered by our detection procedure, averaged over the whole bulge/disk ratio interval. Long-dashed lines represent the fainter envelope of the observed galaxies. The completeness limit is given by the intersection of the latter curve with the brightest segments between simulated ellipticals and spirals. The plume of observed objects with bright magnitudes and with small half-light radius are stars.

case corresponds to an exposure time of $7 \mathrm{~h}$, and defines the complete zone at a magnitude of $K \mathrm{~s}=23.8$.

\section{Colour estimate}

As described in Sect. 2, the GOODS data span a wide range of image qualities, both in terms of resolution/sampling and in terms of limiting depth. In this case, it is difficult to design the optimal strategy. On the one hand, one would like to make full use of the spatial and morphological information contained in the highest quality images, limiting the loss of information occurring when lower resolution images are included as much as possible. This requirement would call for restricting the area over which photometry is done. On the other hand, an unbiased estimate of the colours is essential for most of the scientific application of the GOODS dataset (e.g. photometric redshifts or analysis of the multiwavelength spectral distribution). Since most of the detected objects are galaxies that may exhibit colour gradients due to a change in the morphological properties across the wavelengths, one would like to ensure that a properly wide area is taken for each object. Finally, an optimal $\mathrm{S} / \mathrm{N}$ is required to improve the accuracy of photometric redshifts.

To match these discordant requirements as closely as possible, we adopted two different techniques to accurately measure colours, which we adopt in the ACS and in the groundbased images, respectively. These methods are described in this section.

\subsection{Colours in the ACS images}

Colours in the ACS images were computed by using the isophotal magnitudes as obtained by SExtractor in "dual image" mode. This procedure ensures that the photometry is computed at the different wavelengths in the same physical region, defined by the isophotal area in the "detection" image (here, the $z$ band). In the high resolution ACS images, the isophotal area follows the apparent size of the objects and is relatively insensitive to the effects of nearby contaminants. For these reasons, it has also been used by a number of works dealing with similar data set (Labbé et al. 2003; Vanzella et al. 2001; Cimatti et al. 2002).

However, we also explored alternative solutions, all based on the "dual image" mode of SExtractor. A fixed aperture that is commonly used in the ground-based photometry of faint objects is inapplicable for the large dynamical range of the GOODS images: the typical isophotal radii range from 2 to 20 pixels, but can be as large as 150 pixels.

"Kron" magnitudes ("MAG_AUTO” in SExtractor slang) are typically estimated on areas that are larger than isophotal ones, and might be less sensitive to colour gradients. We verified on bright, as well as on faint, isolated objects that they provide colours that are (on average) identical to isophotal ones. However, they may be contaminated by neighbouring objects and typically have a lower $\mathrm{S} / \mathrm{N}$ than isophotal, since they include more pixels of low $\mathrm{S} / \mathrm{N}$.

We also attempted to use an "optimal" circular aperture, defined as the aperture which maximises the $\mathrm{S} / \mathrm{N}$ of each object in the detection image. Although promising, we verified that it is difficult to provide a robust estimate of such an aperture, since a nearby galaxy may easily contaminate the automatic 
estimate of the $\mathrm{S} / \mathrm{N}$ of faint galaxies. Eventually, we compared the quality of the photometric redshifts obtained with different colour estimators, applied to objects with a spectroscopic redshift, finding that isophotal colours provide a slightly better result than other choices. On the basis of these results, we adopted isophotal colours for all the objects in the sample, on all the ACS images. For ground-based images, we developed a more complex technique that we discuss below.

\subsection{PSF-matched colours in ground-based images}

To measure colours between ACS and ground-based images, we specifically developed and adopted a "PSF-matching" code designed to work especially for faint galaxies, which makes it possible to accurately measure colours in relatively crowded fields, while making full use of the spatial and morphological information contained in the highest quality images.

The technique that we adopted has been introduced for the first time by the Stony-Brook group to optimise the analysis of the $J, H$, and $K$ images of the Hubble Deep Field North. The method is described in Fernandez-Soto et al. (1999), and the catalog obtained has been used in several scientific analyses of the HDFN, both by the Stony-Brook group (e.g. Lanzetta et al. 1999; Phillipps et al. 2000), as well as by several other groups. More recently, a conceptually similar method has been developed by Papovich et al. (2001) to deal with similar problems existing in the first release of GOODS data set.

\subsubsection{The ConvPhot algorithm}

A full description of our code that we name ConvPhot is beyond the aim of the present work. Since we make it publicly available, we refer the reader to its accompanying manual (De Santis et al., in preparation) for a full description. We recall the basic principle here and highlight the possible systematic effects, the strategy that we adopted to minimise them, and the validation tests we performed.

Conceptually, the method is quite straitforward and can be better understood by looking at Fig. 6, where we plot the case of two objects that are clearly detected in the "detection image" but severely blended in the "measure" one. The procedure followed by ConvPhot consists of the following steps:

a) Each object is extracted from the "detection image", making use of the parameters and of the isophotal area defined by SExtractor. Since the latter typically underestimates the actual object size, the SExtractor isophotal area is expanded by an amount that is proportional to the object size.

b) Each object is individually filtered to match the "measure" PSF and normalised to unit total flux: we refer to the resulting thumbnails as the "model profiles" of the objects.

c) The intensity of each "model" object is then scaled in order to match the intensity of the object in the "measure" image. The free parameter for this scaling $\left(F_{i}\right)$ is computed with an $\chi^{2}$ minimisation over all the pixels of the images, and all objects are fitted simultaneously to take the effects of blending into account between nearby objects. Although the number of free parameters that is equal to the number of
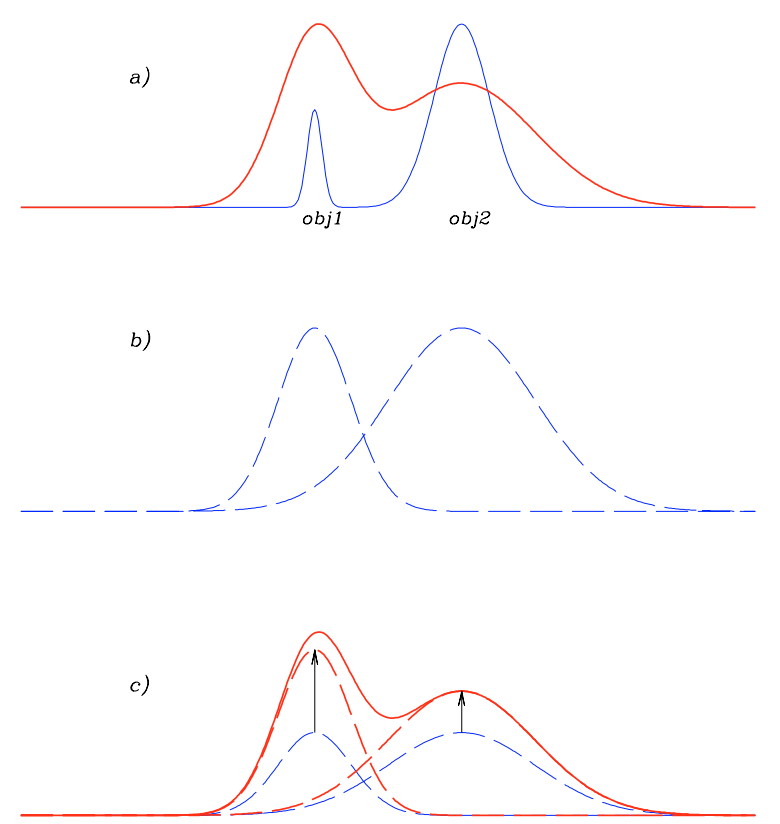

Fig. 6. A schematic representation of the ConvPhot algorithm. a) Two objects are clearly detected and separated in the high resolution detection image (blue, solid-thin line). The same two objects are blended in the low resolution "measure" image (red, solid-thick line) and have quite different colours. b) The two objects are isolated in the high resolution detection image and are individually smoothed to the PSF of the measure image to obtain the "model" images. c) The intensity of each object is scaled to match the global profile of the measure image. The scaling factors are found with a global $\chi^{2}$ minimisation over the whole object pixels.

identified objects is quite large, the resulting linear system is very sparse and can be efficiently solved using standard numerical techniques.

\subsubsection{Systematics in the PSF-matching}

As can be appreciated from the example plotted in Fig. 6, the main advantage of the method is that it relies on the accurate spatial and morphological information contained in the "detection" image to measure colours in relatively crowded fields, even in the case where the colours of blended objects are markedly different.

Still, the method relies on a few assumptions that must be well understood and taken into account. In particular, morphology and positions of the objects should not change significantly between the two bandwidths. Also, the depth and central bandpass of the "detection" image must ensure that most of the objects detected in the "measure" image are contained in the catalog. The objects that are deeply blended in the measure image should be separated well on the detection image.

In practice, it is unlikely that all these conditions are satisfied in real cases. In the case of the match between ACS and ground-based $K$ s images, for instance, very red objects may be detected in the $K$ s band with no counterpart in the optical images, and some morphological change is expected due to the increasing contribution of the bulge in the near-IR bands. Also, in the case where the pixel size of the "measure" image (i.e. 
ISAAC or VIMOS) is much larger than in the "detection" one (ACS $z$ in our case), the actual limit intrinsic accuracy in image aligning may lead to systematic errors.

To characterise and deal with these possible uncertainties, we included several options and fine-tuning parameters in the code to minimise the systematics involved, and used extensive simulations to choose them in an optimal way. We briefly describe them here, referring to a separate paper for a full description.

\section{Missed flux in the outer area}

The Segmentation image provided by SExtractor is usually smaller than the actual object size, since it is limited to the threshold used to detect that source. As a result, the object profile and total flux are incorrectly recovered, which may result in a systematic bias in the fitted colours.

To minimise this effect, we expanded the segmentation produced by SExtractor by an amount that is proportional to the object size, with a minimum area. A minimum value for the area of extended segmentation is useful in the ACS images of the GOODS-CDFS, where extended but low-surface brightness objects are detected only due to the bright nucleus, and the isophotal area is limited only to the brighter knots. For the GOODS-CDFS $z$-band image we dilate the resulting segmentation such that the objects are doubled in linear dimensions, preserving their shape, and in the case of very small objects, the area is set to an equivalent 1 arcsec (diameter) circular aperture.

Blended sources in the detection image

The ConvPhot algorithm is ideally suited to photometry of blended objects on the measure image, but requires that the same objects in the detection image should be well defined and their profiles not be distorted by noise. In the practical case, even in the GOODS-ACS images, there are galaxies blended in the ACS images or faint objects, brighter than the detection limit but still affected by noise in their shape/profile. To overcome this problem, we introduced an option in ConvPhot to carry out the fitting procedure only on the central part of the profile: the fit is restricted only to the pixels where the detection image is above a given relative threshold. This ensures that the fit is carried out only where the signal-to-noise of the model (detection image) is high and avoids contamination from nearby sources, which cannot be perfectly modelled in the detection image. A threshold of 0.5 is used for the GOODS dataset, after extensive simulations.

\section{Alignment errors}

The fitting procedure is extremely sensitive to alignment errors. In the simple case the object having a 2-D Gaussian shape, it is easy to show that the resulting flux is systematically underestimated by a factor $f=\exp \left(-\frac{3}{4} \frac{\Delta r^{2}}{\sigma^{2}}\right)$, where $\Delta r$ is the offset in pixel of the central position. When ground-based images are combined to HST images with excellent sampling, this effect is non-negligible. In the case of the GOODS ACS data, for instance, the ACS pixel size is $0.03^{\prime \prime}$, which is often smaller than the residuals of the alignment of IR ground-based images. For an alignment error of 1 pixel (in the detection image), a figure that can be quite typical or even optimal when combining ground-based and HST images, the resulting underestimate may be about $3 \%$.
As a first way out, we included an option in ConvPhot to re-centre any object before minimisation. In this case, the centre of each object is internally computed in the model, as well as in the measure image, and the measure is re-centered to the model image before minimisation. Since the centre determination may be noisy for faint objects, the user can set a limit on the $\mathrm{S} / \mathrm{N}$ of the objects (in each image) for this operation. We use the re-centering options only for WFI $U$ band images, only for sources with $S / N \geq 15$ both in model and measure images.

\section{Variable FWHM or object profile}

Another source of uncertainty may result from a variation in the object profile from the detection to the measure image, i.e. when the profile in the model image is markedly different from the real profile in the measure image, after the smoothing with the PSF transformation kernel. This can be due to either a physical change of the object profile (as due, for instance, to a more prominent bulge in the IR) or to an incorrect estimate of the PSF transformation kernel. In this case, the resulting flux can therefore be either under- or over-estimated, depending on the sign of the error in the PSF estimate. An error of $10 \%$ in the object $F W H M$ will result in a 5\% error in the output flux.

The small systematic effects that have been described above, or others resulting from different sources, can be efficiently corrected by taking the flux in residual image into account. For this purpose, we included an option in the code to compute the total residual flux contained in the segmentation area of the original frame. This ensures the systematics of colour estimations to lower down, as shown in the next paragraph and in the ConvPhot paper (De Santis et al., in preparation).

\subsubsection{Validation tests}

We performed several validation tests on the ConvPhot code, during the debugging phase to estimate the efficiency in the correction for systematics. The most obvious involved the use of simulated images, with a range of luminosities, PSF, and morphologies, by which we verified that the code is computationally correct (De Santis et al., in preparation).

Simulations, however, may not fully reproduce the complexity of real objects and data. To obtain a more stringent and independent test, we made use of the $z$ band FORS image of the CDFS obtained within the K20 survey (Cimatti et al. 2002). Here, we used ConvPhot to obtain a new estimate of the $z_{\mathrm{ACS}}-z_{\mathrm{FORS}}$ colour in the FORS image of the K20. Since the two $z$ filters are fairly similar, all objects should have a null colour, barring variable objects. In practice a small offset $(0.035 \mathrm{mag})$ between the two bands persists still even at bright magnitudes, probably due to a different response for the ACS and FORS instruments. We limit the comparison to $z \leq 24.75$, the limit of FORS $z$ band at $S / N=10$. Due to the brighter magnitude limit of the FORS image, the error in the $z_{\mathrm{ACS}}-z_{\mathrm{FORS}}$ colour is dominated by the error on the FORS $z$-band magnitude estimate. The results of this comparison are shown in Fig. 7, and show that the ConvPhot software is not biased in the colour determination. 

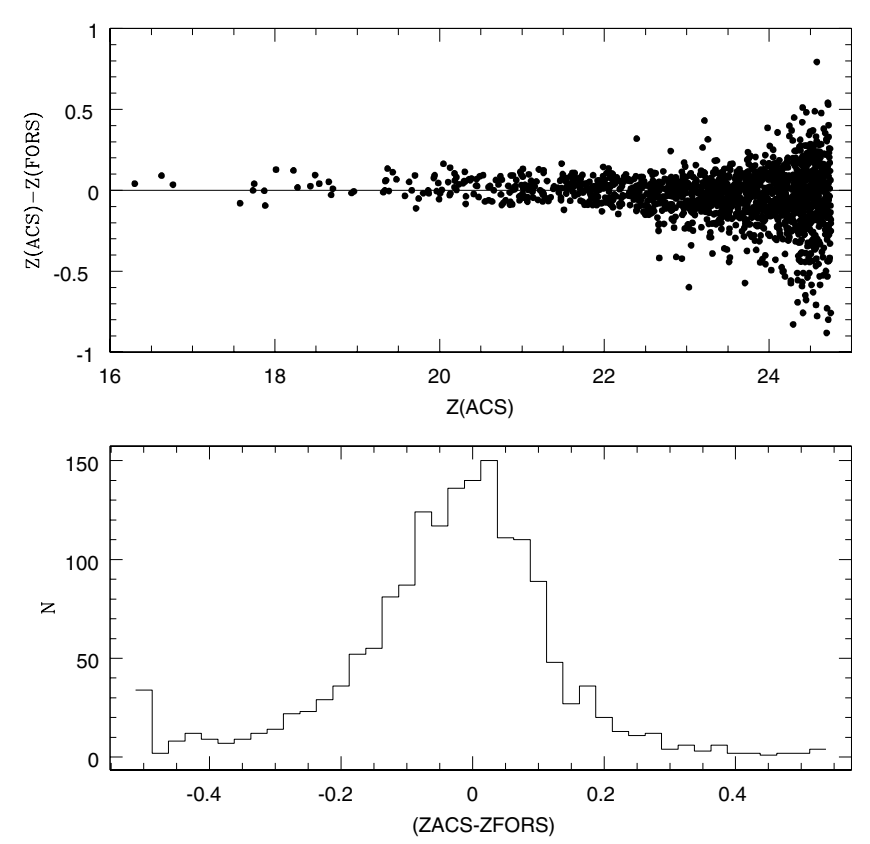

Fig. 7. The $z_{\mathrm{ACS}}-z_{\mathrm{FORS}}$ colour as a function of the magnitude of the objects detected on the K20 survey (upper panel). The histogram of this colour (lower panel) shows that the ConvPhot magnitude determination is not biased and is compatible with aperture photometry.

\subsection{The final catalogs}

The direct output of a ConvPhot run is the scaling parameter $F_{i}$ for each object. Since the model profile $P_{i}$ for each object is normalised to unit flux, the resulting total magnitude in the measure image is simply $-2.5 \log \left(F_{i}\right)+Z P_{m}$, where $Z P_{m}$ is the zeropoint of the measure image itself. Based on our tests, we concluded that this total magnitude is a reliable measure of the actual total flux of the objects, somewhat less prone to systematic effects than the Kron magnitudes computed by SExtractor. However, these total magnitudes can hardly be compared with the SExtractor magnitudes of the detection image, so that reliable colours cannot be obtained directly. To this end, we used the total flux $D_{i}$ measured by ConvPhot itself in the detection image and used it to normalise the object profile $P_{i}$. The resulting flux ratio is therefore Flux(measure $) /$ Flux (Detection $)=$ $F_{i} / D_{i} \times 10^{-0.4\left(Z P_{\mathrm{m}}-Z P_{\mathrm{d}}\right)}$, where $Z P_{\mathrm{d}}$ is the zeropoint of the $d e-$ tection image. This flux ratio, or the equivalent magnitude colour, $m_{\text {measure }}-m_{\text {detection }}=-2.5 \log \left(F_{i} / D_{i}\right)+Z P_{\mathrm{m}}-Z P_{\mathrm{d}}$ is the final colour that we used in the following. All colours were finally normalised to the total $z$ magnitude to obtain selfconsistent magnitudes at all wavelengths.

As stated above, the final colours were obtained with ConvPhot. For the $J, H$, and $K$ s images, we resampled and aligned each individual VLT-ISAAC image to the ACS $z$ mosaic that we used for object detection, and used the $z$ image, smoothed with the appropriate convolution kernel, as the "model" image. In the case of the $U_{35}, U_{38}$, and $U_{\text {VIMOS }}$ images, we followed the same procedure, after cutting the original $U$ images into smaller pieces (since ConvPhot can operate only with small size images, due to memory limitations), but using the ACS $B$ image, smoothed with the appropriate convolution kernel, as a "model" to minimise the effects of wavelength-dependent morphologies. Because of the geometry of IR images, several objects were detected in more than one IR pointing. In these cases, an optimally weighted average was used to estimate the final colour. In all cases, we conservatively dilated the "Segmentation" image by a factor of 4 , with a minimum area after the dilate of 800 pixels (corresponding to a PSF with 1 arcsec of diameter), and applied a fitting threshold of 0.5 to minimise the effects of nearby contaminants. For ISAAC and VIMOS images, we did not use the centering option, while for the $U_{35}$ and $U_{38}$ photometry we applied a centering with ConvPhot for objects with $S / N \geq 15$.

The same technique was applied to the Spitzer images. We are aware that the properties of the PSF of the IRAC instrument is not fully characterised ( $F W H M$ variation across the FoV), and the much lower image quality (the FWHM ranges from 1.6 to 2.0 arcsec, see Table 1) makes the whole analysis more difficult and uncertain. For this reason, we regard the colour estimate in the Spitzer bands as more uncertain than in the other wavelengths, and we plan to address it in more detail in a future paper where we will also deal with the Spitzer-selected samples.

For the Spitzer bands, we used ConvPhot to carry out the photometric analysis of the GOODS sources detected in the $z$ band. We dilated the "Segmentation" image by a factor of 4 , with a minimum area after the dilate of 800 pixels, and applied a fitting threshold of 0.5 to minimise the effects of nearby contaminants. For IRAC images, we haven't used the centering option.

Figure 8 shows the colours obtained for all galaxies with spectroscopic redshift in our sample (the sources of the spectroscopic data adopted are listed in Sect. 6) in a few selected bands. The corresponding range obtained from the Bruzual \& Charlot (2003) program is also shown for two extreme models, a very young, star-forming model, and a maximally old model with a short e-folding star-formation timescale. Most of the objects lie within these boundaries at the different redshifts, providing a reassuring quality check on the overall photometry. We verified that such an agreement still holds in the other bands.

\section{The Ks-selected catalog}

The catalog produced using the $z$ band of ACS as the detection image is not complete in the $K \mathrm{~s}$ band magnitude. In principle, this could be obtained by using the $K$ s images as detection images, and computing colours in the other bands. The catalog obtained, however, would not benefit from the higher spatial resolution of the ACS images, and the two catalogs would not be homogeneous. To prevent this, we followed a different approach of identifying the objects missed in the $z$-selected catalog in the so-called "Drop" images produced by ConvPhot in the $K \mathrm{~s}$ band, i.e. the residuals of the fitting procedure.

An example is shown in Fig. 9, which shows that there are objects that are not detected in the $z$ band but bright in the $K \mathrm{~s}$ band. To identify all these missed objects, we first carried out a run with ConvPhot with a dilate parameter equal to 0 and using small smoothing kernels in order to fit only the central part of the $z$ detected objects and not to avoid missing $K$ s bright galaxies close to $z$ detected objects. In this way the Drop 


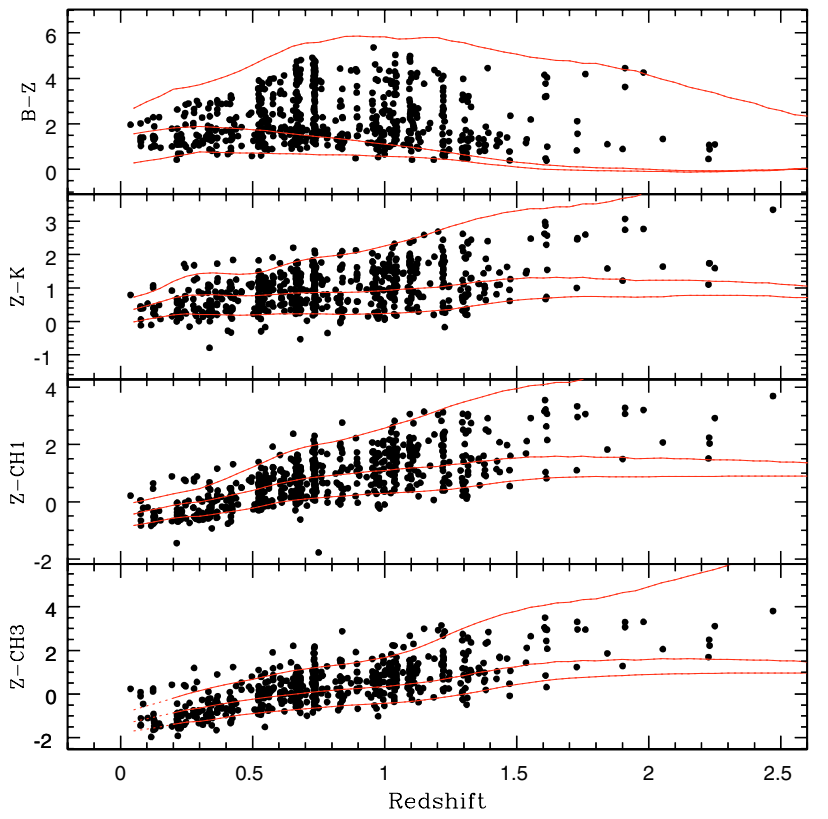

Fig. 8. Observed colours as a function of redshift for the objects with spectroscopic redshifts in the GOODS-S field. The solid lines are (from top to bottom) the colours predicted by the Bruzual \& Charlot (2003) code for the following cases: (i) a maximally old model, obtained assuming an exponential history of star-formation with timescale $0.1 \mathrm{Gyr}$ started at $z=10$ with metallicity $Z=2.5 Z_{\odot}$; (ii) a similar model but with an exponential timescale of $5 \mathrm{Gyr}$ and an actively star-forming and non-evolving model with a constant starformation rate and age of $1 \mathrm{Gyr}$. Dotted lines represent the redshift range in which the colours are not reliable due to the incomplete modelling of dust emission at $\lambda \geq 5.5 \mu \mathrm{m}$ rest frame (see Sect. 7).

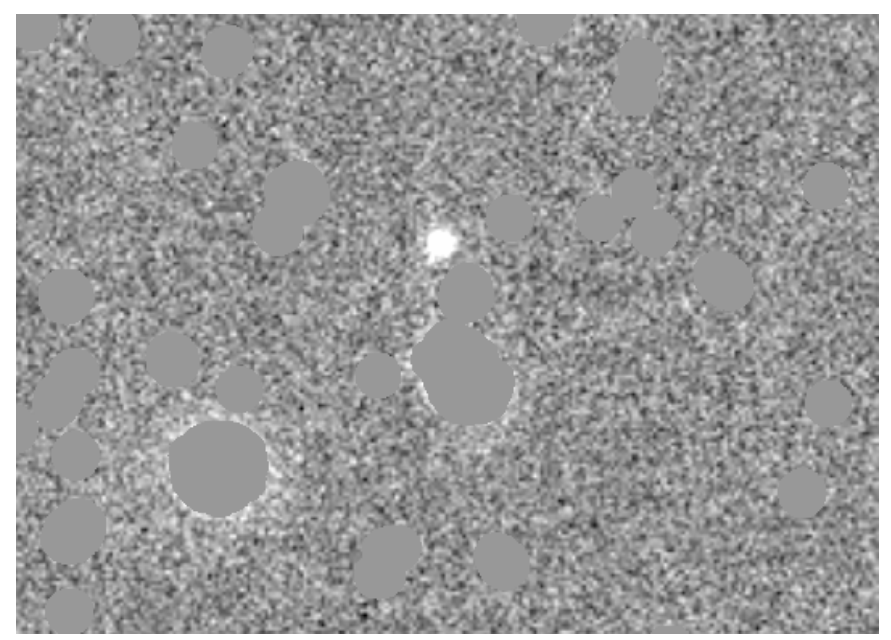

Fig. 9. A so-called "Drop" image produced by ConvPhot in the $K \mathrm{~s}$ band. The name derives from the fact that each $z$-band detected object that is fitted by ConvPhot is multiplied by zero (drop) in order to leave only those $K$ s bright galaxies not detected in the $z$ band.

images have a minimal area covered by "drops", and the completeness of the $K$ s selected objects is enhanced.

We ran SExtractor on the $K \mathrm{~s}$ "Drop" images, producing a complete catalog, whose depth will follow the exposure maps described above. On the basis of the simulations described above, it is typically complete down to $K \mathrm{~s}=23.8 \mathrm{mag}$ ( $\mathrm{AB}$ ) at the $90 \%$ completeness level. As total $K$ s band magnitude, we used the BEST magnitude of SExtractor.

The extraction of the colour information for these $K \mathrm{~s}$ selected objects is not trivial. We calculated the colours in an isophotal area defined by the dilated segmentation of the $K$ s band objects. In order to provide an unbiased estimate for the colours, we resampled the ACS images to the $K$ s resolution using the convolution kernel adopted by ConvPhot. The total magnitude in these bands is computed using the $K \mathrm{~s}$ as reference and the colour term as an additive effect: $B_{\text {tot }}=$ $K \mathrm{~s}_{\text {tot }}+(B-K \mathrm{~s})_{\text {iso }}$, where $(B-K \mathrm{~s})_{\text {iso }}$ is the colour computed in the "extended" isophotal area. For the other bands ( $U$ and IRAC), where the $F W H M$ is larger than $K$ s, we resampled the $K$ s band image to match the measure image PSF.

At the end we detected 196 objects not present in the $z$ selected catalog. The nature of these objects is typically twofold: they can be low-surface brightness galaxies in the ACS $z$ band images, extended objects that escape from detection criterion in $z$, but with bright total magnitudes detected in the $K$ s band because of a lucky combination of pixel-size and seeing effects $(151,77 \%)$. The other type is constituted by objects that are not detected in the ACS image because they are really $z$-dropout objects, basically EROs at $z \geq 1-2(42,21 \%)$. Only three objects are not part of these two categories: bright objects in the $z$ and $K \mathrm{~s}$ bands that are not detected in $z$ because they are on a spike of a bright star ( 2 objects) or in the halo of a bright and extended galaxy (1). We recover them from the $K$ s Drop images.

We merged the $z$-selected catalog with the Drop $K$ s selected objects in order to build a total catalog of objects that can be considered complete down to $z \simeq 26.0$ and $K \mathrm{~s} \simeq 24.0$ simultaneously. In the catalog, $I D \leq 20000$ indicates $z$-detected objects, while $I D \geq 30000$ points to "drop" $K$ s selected objects. We also computed, as described in Sect. 2.4, the limiting magnitudes (at $1 \sigma$ and in 1 sq. arcsec area) in $z$ and $K$ s bands for the $K$ s detected objects, in order to have the same quantities of the $z$-detected catalog to ensure a given homogeneity to the total catalog. At the moment there is no spectroscopic information for the $K$ s drop detected galaxies, since they are not bright enough (in surface brightness limited samples) in the optical bands or not present in previous ACS-based catalogs used for spectroscopic identifications.

\section{The spectroscopic catalog}

The GOODS-CDFS field has been the target of a number of spectroscopic surveys that paved this area with unprecedented accuracy. The various catalogs surveyed this field for different reasons: the COMBO-17 survey (Wolf et al. 2001) needed spectra to calibrate their photometric redshifts over a wide and relatively shallow area; the CXO survey (Szokoly et al. 2004) is a spectroscopic follow-up of X-ray sources in the Megaseconds CDFS; and the K20 survey (Mignoli et al. 2005) provides the spectra for 327 sources with $K \leq 20$ (Vega). After the public release of the ACS GOODS images, two spectroscopic surveys were released: the GOODS V1.0 spectroscopy (Vanzella et al. 2005) and the VVDS survey (Le Fevre et al. 2004) with the aim of providing a comprehensive census of bright objects in 
the GOODS field. Recently the ESO-GOODS team released a Master ${ }^{3}$ catalog of spectra in the GOODS-CDFS area, which summaries all the redshift information of the previously cited catalogs, with additional redshifts from sparse surveys.

The information provided by these surveys is basically coordinates and redshifts. The classification (Star, galaxy, or AGN) and a quality flag for the spectrum are provided by all of the catalogs, but are not present in CXO and Master catalogs. The quality flags of the various catalogs are not homogeneous, so we tried to define a homogeneous classification for all the catalogs. For the K20 catalog, the quality of the redshifts is $q_{\mathrm{K} 20}=1$ for the secure redshifts and $q_{\mathrm{K} 20}=0$ for the uncertain ones. For the GOODS spectroscopy, the quality flag goes from $\mathrm{A}$ to $\mathrm{C}$, towards a decreasing $\mathrm{S} / \mathrm{N}$ of the spectra. The VVDS uses a reverse scheme when compared to our way of quantifying the quality of the spectra, with $q_{\mathrm{VVDS}}=4$ for the highest quality to $q_{\mathrm{VVDS}}=1$ for the lowest one. We define four classes of quality flags, from 0 to 3 , where $q z=0$ indicates the best spectra, with secure identification and $q z=3$ the most noisy spectra. For the other spectroscopic catalogs, where the quality flag is not available, a $q z=1$ is defined a priori. Only for the K20 and GOODS spectroscopic surveys is there the distinction between early or emission line galaxy (or a combined spectrum). For the other catalogs, the classification is not homogeneous and is not subdivided in finer classifications such as early, emission line, or composite spectra. In order to have a simple classification scheme, we divided all the objects with spectroscopic information in these classes: STAR, AGN, GALAXY, EARLY, EMISSION, or COMPOSITE. The classification EARLY, EMISSION, or COMPOSITE only comes from the K20 and GOODS spectroscopic surveys.

Since in several cases there are multiple identifications for the same object, we cross-correlated the catalogs to check for inconsistencies; if there are two different identifications for the same object, we take the identification with best quality flag. In the GOODS ACS area there are currently 1068 known spectroscopic redshifts: Fig. 10 shows the redshift distribution of these objects, in which three distinct groups/z-peak are visible, corresponding to different groups or sheets of galaxies. There are in total 928 galaxies, 72 stars, 68 AGNs, and QSOs that are already known. In the galaxy sub-sample, only 668 have a secure redshift, while the remaining are not secure. For 428 galaxies the finer classification (early-emission line) is available, where "only" 94 are classified as early and 67 as composite spectrum.

The identification of the spectroscopic catalog with objects in the ACS images is not trivial, due to the finest resolution of HST compared to ground-based images, where close-by objects are merged into a single blob, or because there is a coordinate mismatch, especially for the K20 and CXO surveys, based on imaging material precedent to the ACS one. To overcome this problem/issue, we carried out a cross correlation in right ascension and declination between the photometric data and the spectroscopic catalog with a relatively large matching radius (1.2 arcsec) and divided the objects into two groups: isolated objects, with a unique identification and blended or

\footnotetext{
${ }^{3}$ http://www.eso.org/science/goods/spectroscopy/ CDFS_Mastercat/
}
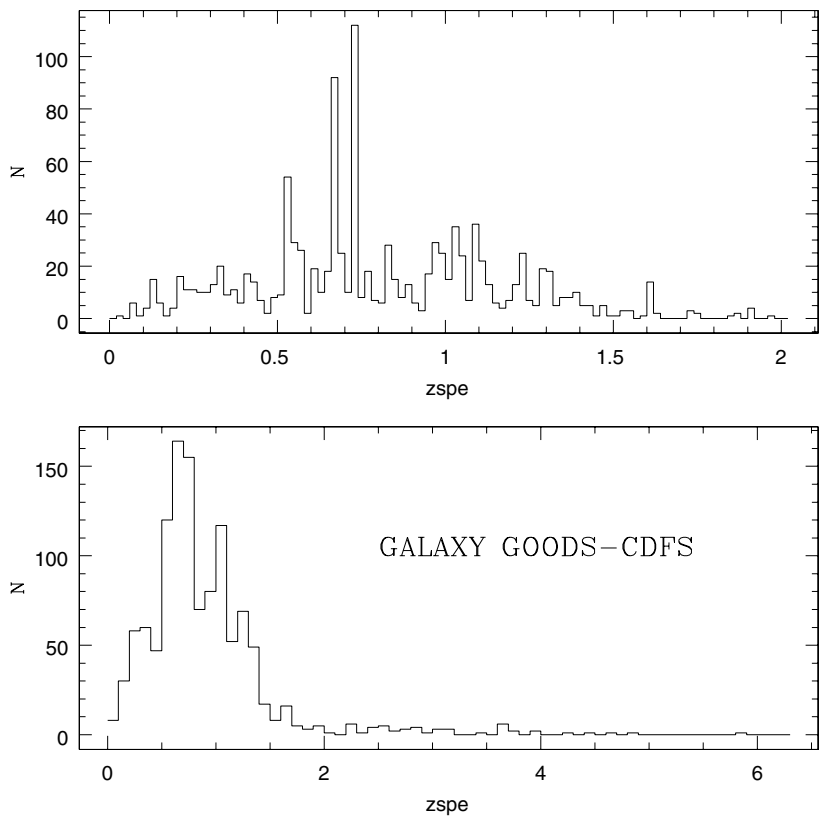

Fig. 10. The histogram of spectroscopic redshifts for galaxies in the GOODS-CDFS field. Three peaks in redshifts show the presence of large-scale structures like groups or sheet/wall of galaxies.

ambiguous objects, where more than one photometric ID is associated with a spectroscopic data. In the last case the association is checked by eye and decided also using the magnitude/colour information.

Overall, less than 10 percent of the GOODS catalog has any spectroscopic information (1068 out of 14847 ). For the remaining objects, having a high photometric quality and a wide wavelength sampling of the SEDs, we chose the approach of the photometric (Sect. 7) and neural network redshifts (Vanzella et al., in preparation).

\subsection{Star-Galaxy separation}

We distinguished galaxies from stars and AGNs using morphological and photometric information, when spectroscopic data were not available. First, point-like sources were selected using the star/galaxy separation flag $(\mathrm{s} / \mathrm{g})$ provided by SExtractor in the $z$ band. We tuned the selection on already known spectroscopic stars: objects with magnitude $z \leq 20$ and $s / g \geq 0.80$, $20<z \leq 23$ and $s / g \geq 0.96$ or $23<z \leq 24$ and $s / g \geq 0.98$ were considered possible AGNs or stars. We used photometric information to check this criterion, using in particular the "BzK" colour criteria of Daddi et al. (2004). Figure 11 shows the positions of AGNs, galaxies, and stars in the $B-z$ versus $z-K s$ two-colour plot. Typically, the point-like objects selected with the SExtractor parameter were found to lie on the lower side of the diagram, where stars are expected to be found. Other objects located in (or nearby) this region, but not selected morphologically, were visually inspected. Most of them turned out to be extended, low-surface brightness galaxies, most likely at very low redshift (and hence with similar colour to Galactic stars). The remaining unresolved objects were classified as stars. 


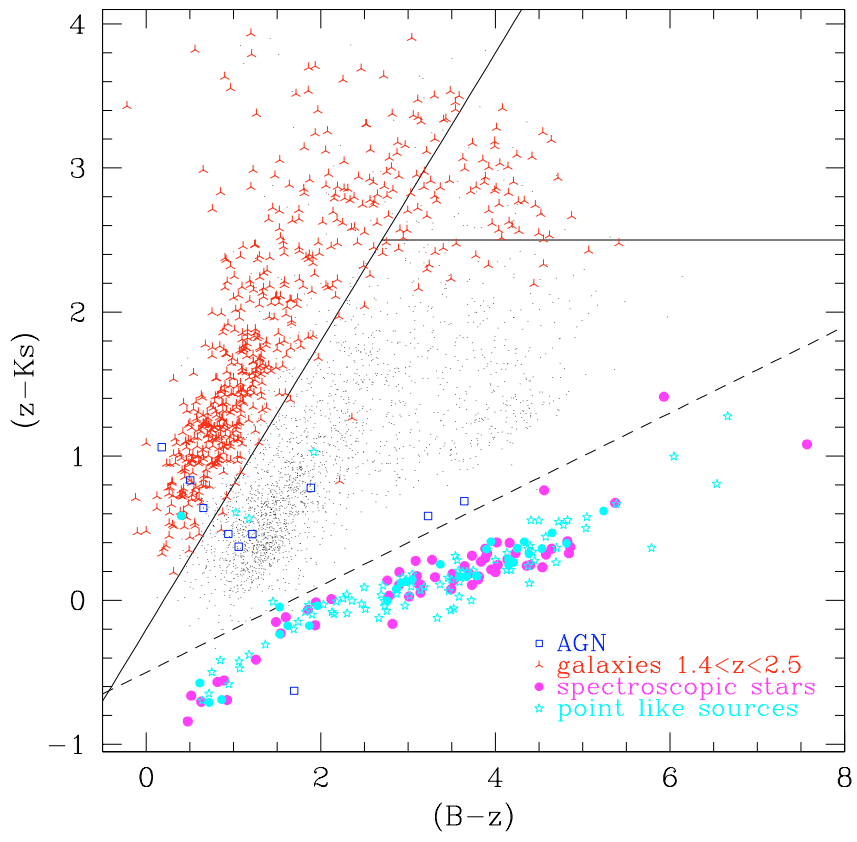

Fig. 11. The $B-z$ versus $z-K$ s colours for objects in the GOODS south. Small dots are galaxies with redshifts $z \leq 1.4$ or $z \geq 2.5$, squares are spectroscopically known AGNs, arrows indicate galaxies with $1.4<z<2.5$ (spectroscopic and photometric redshifts), filled points are spectroscopically known stars, while stars represent objects with point-like morphology according to SExtractor. Solid lines indicate the BzK criterion to isolate star-forming (upper left part of the diagram) and passively evolving galaxies (upper right part of the diagram) at $1.4<z<2.5$. Objects below the dashed line are stars, according to Daddi et al. (2004).

\section{Photometric redshifts}

On the multicolour catalog described above we applied our photometric redshift technique, which was described in detail in Giallongo et al. (1998) and Fontana et al. (2000) (F00 hereafter) which proved to be extremely successful in the HDFN/S (Fontana et al. 2003) and in the K20 data sets (Cimatti et al. 2002; Fontana et al. 2004).

In essence, a spectral library of galaxies at arbitrary redshifts is computed and a $\chi^{2}$-minimisation procedure is applied to find the best-fitting spectral template to the observed colours. For each template $t$ at any redshift $z$ we first minimise

$\chi_{t, z}^{2}=\sum_{i}\left[\frac{F_{\text {observed }, i}-s \cdot F_{\text {template }, i}}{\sigma_{i}}\right]^{2}$

with respect to the scaling factor $s$ where $F_{\text {observed, } i \text { is the flux }}$ observed in a given filter $i, \sigma_{i}$ is its uncertainty, $F_{\text {template, } i}$ is the flux of the template in the same filter, and the sum is over the filters used. We then identify the best-fitting solution with the lowest $\chi_{t, z}^{2}$. We refer to the discussion in F00 for its detailed handling of the non-detection at faint fluxes. The scaling factor $s$ is therefore applied to the input parameters of the bestfitting spectrum to compute all the rest-frame quantities, such as absolute magnitudes or stellar masses.

We tested different choices for the spectral libraries, using both empirical templates (based on the Coleman et al. data set), as well as on synthetic models, namely the
Bruzual \& Charlot (2003) and the PEGASE 2.0 (Fioc \& Rocca-Volmerange 1997). We found that the use of synthetic models results in a better agreement with the spectroscopic sample available and that, in particular, the choice of the PEGASE 2.0 models minimises both the rms and the number of outliers with respect to the $\mathrm{BC} 03$ library. In this paper, we briefly describe the results obtained with the PEGASE 2.0 spectral library to provide a quantitative description of the quality of our results, and we defer to a forthcoming paper a more accurate comparison of the performances of these libraries, which compares these recipes also with the alternative approach of neural networks (Vanzella et al. 2004).

An application of the PEGASE 2.0 code to photometric redshifts has been presented by Le Borgne \& Rocca-Volmerange (2002) on the HDFN dataset. A major advantage of the PEGASE 2.0 code is that it allows to follow the metallicity evolution explicitly, also including a selfconsistent treatment of dust extinction and nebular emission. We adopt the same parameter grid as in Cimatti et al. 2002 and Fontana et al. 2003 which is very close to the Le Borgne \& Rocca-Volmerange (2002) prescriptions. In detail, we parameterize the star-formation history by two $e$-folding starformation time-scales, one $\left(\tau_{\mathrm{g}}\right)$ describing the time-scale for the gas infall on the galaxy and the other $\left(\tau_{*}\right)$ the efficiency of gas to star conversion. By tuning the two time-scales it is possible to reproduce a wide range of spectral templates from early types (by using small values of $\tau_{\mathrm{g}}$ and $\tau_{*}$ ) to late. For the earliest spectral type, a stellar wind is also assumed to block any star-formation activity at an age $t_{\text {wind }}$. Dust content is followed over the galaxy history as a function of the on-going star-formation rate, and an appropriate average over possible orientations is computed. The range of values for $\tau_{\mathrm{g}}, \tau_{*}, t_{\mathrm{wind}}$ and the extinction geometry are listed in Table 4 for a Rana \& Basu (1992) IMF. For all these models we have adopted a primordial initial metallicity. Another differences in our approach compared to Le Borgne \& Rocca-Volmerange (2002) are that we do not apply any constraint on the galaxy ages (apart from those set by the Hubble time at each $z$ ) and that we have allowed for a finer time sampling in the galaxy ages by halving the time step with respect to the default value of the PEGASE 2.0 program.

We included the contribution of the nebular emission in the synthetic library, both in the continuum and in the lines, as allowed by PEGASE 2.0. At the Ly $\alpha$ frequency, however, inclusion of the corresponding emission line in star-forming galaxies would introduce a systematic bias, since the observed samples of Lyman break galaxies at $z \simeq 3$ have a wide distribution of observed Ly $\alpha$ equivalent widths, ranging from absorption to emission systems. For this reason, we did not apply the Ly $\alpha$ emission line as a simple tradeoff, and we also removed the strong absorption feature resulting from the stellar features from the synthetic spectrum.

The major difference with the Le Borgne \& Rocca-Volmerange (2002) approach, however, is due to evidence that the spectral library described above is not able to reproduce the reddest objects (EROs or similar) that are typically detected at $z>1$ and that are either passively evolving galaxies or star-forming dusty objects 
Table 4. Parameters used for the PEGASE 2.0 library. Other models were added to reproduce the colours of red galaxies at high redshift, as described in the text.

\begin{tabular}{llll}
\hline \hline$\tau_{g}(\mathrm{Myr})$ & $\tau_{*}$ & $t_{\text {wind }}$ & Extinction type \\
\hline 100 & 100 & 3000 & spheroid \\
100 & 500 & 5000 & spheroid \\
500 & 1500 & - & disk, incl-averaged \\
1000 & 2500 & - & disk, incl-averaged \\
1000 & 5000 & - & disk, incl-averaged \\
2000 & 10000 & - & disk, incl-averaged \\
2000 & 20000 & - & disk, incl-averaged \\
5000 & 20000 & - & disk, incl-averaged \\
\hline
\end{tabular}

(Cimatti et al. 2002) and that we detect in our sample. For this reason, we supplemented the above models with a few templates designed to mimic these objects.

Passively evolving galaxies were extracted from simple truncated models, where a starburst with constant starformation rate is halted after $0.1,0.3,0.6$, or $1 \mathrm{Gyr}$. These models were used only after the truncation age, so they rapidly become redder than the other models. These models are able to reproduce redder colours than the standard models of PEGASE 2.0, particularly in the near and medium infrared part of the SED, thus fitting the ISAAC and IRAC observations better.

Star-forming, dusty objects are instead taken from models with a constant star-formation rate, following a Calzetti extinction curve with $0.5 \leq E(B-V) \leq 1.1$. At each redshift, galaxies are allowed any age that is compatible with the Hubble time at that redshift.

We also added to all models the Lyman series absorption produced by the intergalactic medium (Madau 1995), but we introduce here a simpler parameterization that uses the DA and DB values computed from a compilation of data at $z \leq 4$ and extrapolated at higher $z$ with a simple interpolation to the SDSS data. In particular we use $D A=\left[(1 .+z)^{2.66838} \times\right.$ $\left.10 .^{-2.17281}\right]$ and $D B=D A^{-0.3576+1} \times 10^{-0.03616}$ at $z \leq 4$, and $D A=0.187 z-0.247$ and $D B=1.178 z-0.133$ at $4.3<z<6.3$, with $D A=D B=1$ at greater $z$.

The most important difference with respect to previous papers arises from the inclusion of the Spitzer bands, which (at low and intermediate redshifts) extend the observation to the spectral regions longer than $3 \mu \mathrm{m}$ rest frame. The galaxy emission is not dominated by the integrated stellar population at $\lambda \geq 5.5 \mu \mathrm{m}$ (Dale et al. 2005; Lu et al. 2003) but by the different flavours of dust emission, at least in the case of star-forming galaxies that are not included in the spectral libraries considered here. As a simple way out, we modified our code: any photometric band that is contaminated by the dust reprocessed emission above $5.5 \mu \mathrm{m}$ in the rest-frame is ignored in the fit.

We tested our recipe against the large subsample of galaxies with secure spectroscopic redshift, which we compiled as described in the previous section: to this end, we considered 668 galaxies with the spectroscopic flag equal to 0 or 1 . The results are shown in Fig. 12. In its upper panel, we plot the
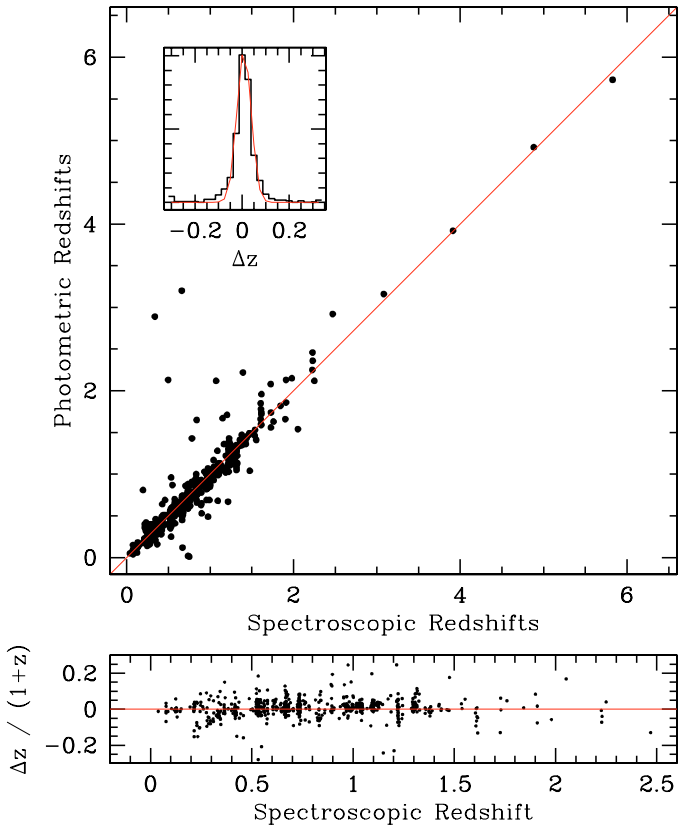

Fig. 12. Upper panel: the relation between the spectroscopic ( $x$-axis) and the photometric ( $y$-axis) redshift on 668 galaxies with accurate spectroscopic redshift. In the inset, the distribution of the absolute scatter $\Delta z=\left(z_{\text {spec }}-z_{\text {phot }}\right)$ is shown and compared with a Gaussian distribution with a standard deviation $\sigma=0.06$ (smooth red curve). Lower panel: relative scatter $\left(z_{\text {spec }}-z_{\text {phot }}\right) /\left(1+z_{\text {spec }}\right)$, restricted to the $z<2$ range and discarding the most discrepant objects in the same sample.

$z_{\text {spec }}-z_{\text {phot }}$ relation to show that we find an excellent agreement between photometric and spectroscopic redshifts over the fully accessible redshift range $0<z<6$, with a very limited number of catastrophic errors. More quantitatively, we plot the distribution of the absolute scatter $\Delta z=\left(z_{\text {spec }}-z_{\text {phot }}\right)$ in the inset: the central part of the distribution is perfectly represented by a Gaussian distribution (smooth red curve) with a standard deviation $\sigma=0.06$ and a small number of outliers. Because of these outliers, the distribution is not Gaussian and the usual rms is not a good indicator of the width of the distribution. If we instead adopt the average absolute scatter, we find $\langle|\Delta z|\rangle=0.08$ and $\langle|\Delta z /(1+z)|\rangle=0.045$ : these values are among the lowest ever obtained with the photometric redshift technique in the redshift interval $0<z<6$. Similarly, the lower panel of Fig. 12 shows the relative scatter $\left(z_{\mathrm{spec}}-z_{\text {phot }}\right) /\left(1+z_{\mathrm{spec}}\right)$, restricted to the $z<2$ range and discarding the most discrepant objects, which has a nearly homogeneous rms of 0.03 . As far as we know, this is the highest precision ever obtained on faint samples spanning such a wide redshift range, although it is still lower than the average SDSS value. Several elements concur to obtain this improvement with respect to similar samples (e.g. Fontana et al. 2003; Mobasher et al. 2004; Vanzella et al. 2004): the larger number of bands, the sophisticated technique adopted for photometry, and the use of PEGASE 2.0 models, which provided slightly better results than other synthetic models or observed templates. We plan to discuss these effects better in a future paper. 
Table 5. Extract of the GOODS-MUSIC web catalog: coordinates, spectroscopy and magnitude limits.

\begin{tabular}{ccccccccccccccc}
\hline \hline ID & $\begin{array}{c}\text { RA } \\
\text { J2000 }\end{array}$ & $\begin{array}{c}\text { Dec } \\
\text { J2000 }\end{array}$ & $z^{a}$ & class $^{b}$ & catalog $^{c}$ & $q z^{d}$ & zphot & POS $^{e}$ & star $^{f}$ & AGN $^{g}$ & zlim $^{h}$ & Kslim $^{i}$ & S/G $^{j}$ \\
\hline 10015 & 53.109565 & -27.788200 & 0.995 & emission & K20 & 0 & 0.995 & 1 & 0 & 0 & 28.044 & 26.982 & 0.030 \\
10016 & 53.166175 & -27.787519 & 1.097 & galaxy & COMBO17 & 0 & 1.097 & 1 & 0 & 0 & 28.026 & 27.008 & 0.000 \\
10017 & 53.124374 & -27.788923 & -1.00 & unknown & unknown & 99 & 0.300 & 1 & 0 & 0 & 28.036 & 26.957 & 0.010 \\
10018 & 53.056438 & -27.788972 & -1.00 & unknown & unknown & 99 & 3.500 & 1 & 0 & 0 & 28.046 & 26.327 & 0.010 \\
10019 & 53.046402 & -27.789001 & -1.00 & unknown & unknown & 99 & 0.280 & 1 & 0 & 0 & 28.051 & 26.136 & 0.740 \\
10020 & 53.065872 & -27.787111 & 0.738 & early & K20 & 0 & 0.738 & 1 & 0 & 0 & 28.036 & 26.326 & 0.030 \\
$\ldots \ldots$. & $\ldots \ldots \ldots$. & $\ldots \ldots \ldots$. & $\ldots \ldots$. & $\ldots \ldots$. & $\ldots \ldots$ & $\ldots$ & $\ldots \ldots$ & $\ldots$ & $\ldots$ & $\ldots$ & $\ldots \ldots$. & $\ldots \ldots$. & $\ldots .$. \\
\hline
\end{tabular}

${ }^{a}$ Spectroscopic redshift $(-1.0=$ not available $)$.

${ }^{b}$ Spectroscopic class (see text).

${ }^{c}$ Reference spectroscopic catalog.

${ }^{d}$ Quality of spectroscopic redshift $(0=$ very good, $1=$ good, $2=$ uncertain, $3=$ bad quality, $99=$ not available).

${ }^{e}$ Position flag $(1$ inside GOODS-MUSIC area, $0=$ outside $)$.

${ }^{f}$ Star flag $(1=$ probable star, $0=$ no star), on the basis of spectroscopy, morphology, and BzK colours (see text).

${ }^{g}$ AGN flag, based only on spectroscopy $(1=$ probable AGN, $0=$ no AGN). A galaxy should have star flag $=0$ and $\mathrm{AGN}$ flag $=0$.

${ }^{h}$ Magnitude limit in the $z$ band in 1 sq. arcsec and at $1 \sigma$.

${ }^{i}$ Magnitude limit in the $K$ s band in 1 sq. $\operatorname{arcsec}$ and at $1 \sigma$.

${ }^{j} \mathrm{Star} /$ Galaxy index of SExtractor.

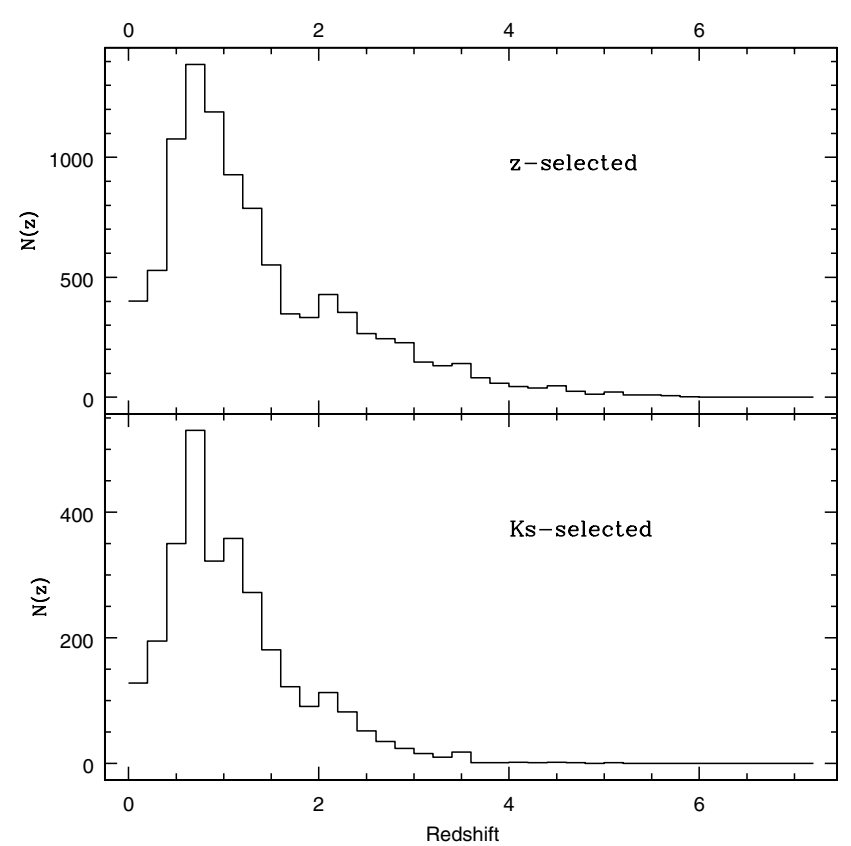

Fig. 13. Upper panel: redshift distribution of 9862 galaxies in the $z$-selected sample. The typical magnitude limit is $z \simeq 26$. Lower panel: redshift distribution of 2931 galaxies in the $K$ s-selected sample. The typical magnitude limit is $K \mathrm{~s} \simeq 23.8$.

The resulting redshift distributions are shown in Fig. 13, both for the 9862 galaxies of the $z$-selected sample and for the 2931 galaxies of the $K$ s-selected one. They were drawn by adopting the photometric redshift for all galaxies except those with a secure spectroscopic one. For the reasons described in Sect. 4, the two samples do not have a unique magnitude limit, but for most of the sample the typical magnitude limits are $K \mathrm{~s} \leq 23.8$ and $z \leq 26$. The two distributions show both a marked peak at $z \simeq 0.7$, which is a well known feature of this field, and a marked decrease at $z>1.5$.

\section{Summary}

In this paper, we have described in detail the procedures adopted to obtain a multiwavelength catalog of the large and deep areas in the GOODS Southern Field covered by the deep near-IR observations obtained with the ESO VLT. The catalog, named GOODS-MUSIC (MUlti-wavelength Southern Infrared Catalog), is made publicly available and we plan to use it in several future scientific analyses. The main features of this work are the following:

- The catalog is based entirely on public data: we included the $F 435 W, F 606 W, F 775 W$ and $F 850 L P$ ACS images in our analysis, along with the $J H K$ s VLT data, the Spitzer data provided by the IRAC instrument $(3.6,4.5,5.8$, and $8.0 \mu \mathrm{m})$, and publicly available $U$-band data from the $2.2 \mathrm{ESO}\left(U_{35}\right.$ and $U_{38}$ ) and VLT-VIMOS ( $U_{\text {VIMOS }}$ ).

- The complications arising from the inhomogeneous coverage and depth of the available images were addressed with great care. The first is that a unique magnitude limit cannot be defined in a given band. For this purpose, we included a careful estimate of the magnitude limits across the detection images $(z$ and $K \mathrm{~s}$ ) to make the extraction of statistically well-defined samples possible. In practice, both the $z$ and the $K$ s-selected catalogs were split into several sub-catalogs with a well-defined magnitude limit, using the bins listed in Table 2 . These catalogs should be used as independent catalogs in all those cases where volume-sensitive statistics have to be applied, as in the case of luminosity densities or luminosity functions. Analyses that are sensitive to the position information (such as clustering) instead requires the full use of the positional information.

- The object detection was first done in the ACS $z$ (i.e. $F 850 L P$ ) image, using a customised version of SExtractor, 
Table 6. Extract of the GOODS-MUSIC web catalog: optical photometry.

\begin{tabular}{|c|c|c|c|c|c|c|c|c|c|c|c|c|c|c|}
\hline ID & $\begin{array}{c}U_{35} \\
\text { mag }\end{array}$ & $\begin{array}{c}U_{38} \\
\text { mag }\end{array}$ & $\begin{array}{c}U_{\mathrm{VIM}} \\
\mathrm{mag}\end{array}$ & $\begin{array}{c}B \\
\text { mag }\end{array}$ & $\begin{array}{c}V \\
\text { mag }\end{array}$ & $\begin{array}{c}i \\
\text { mag }\end{array}$ & $\begin{array}{c}z \\
\text { mag }\end{array}$ & $\begin{array}{l}U_{35} \\
\text { err }\end{array}$ & $\begin{array}{l}U_{38} \\
\text { err }\end{array}$ & $\begin{array}{c}U_{\mathrm{VIM}} \\
\text { err }\end{array}$ & $\begin{array}{c}B \\
\text { err }\end{array}$ & $\begin{array}{c}V \\
\text { err }\end{array}$ & $\begin{array}{c}i \\
\text { err }\end{array}$ & $\begin{array}{c}z \\
\text { err }\end{array}$ \\
\hline 10015 & 23.108 & 23.073 & 99.0 & 23.130 & 22.851 & 22.204 & 21.905 & 0.017 & 0.044 & 99.0 & 0.009 & 0.006 & 0.007 & 0.006 \\
\hline 10016 & 23.085 & 22.954 & 99.0 & 23.029 & 22.618 & 21.938 & 21.425 & 0.031 & 0.070 & 99.0 & 0.017 & 0.010 & 0.011 & 0.008 \\
\hline 10017 & 26.356 & -25.417 & 99.0 & 28.096 & 26.397 & 25.339 & 24.992 & 0.965 & 1.085 & 99.0 & 0.207 & 0.084 & 0.084 & 0.067 \\
\hline 10018 & 26.806 & 24.918 & 99.0 & 27.257 & 25.828 & 25.444 & 25.523 & 0.820 & 0.477 & 99.0 & 0.274 & 0.046 & 0.062 & 0.086 \\
\hline 10019 & 27.843 & -26.546 & 99.0 & 29.091 & 26.807 & 26.547 & 26.550 & 1.014 & 1.085 & 99.0 & 0.630 & 0.076 & 0.107 & 0.133 \\
\hline 10020 & 24.893 & 24.945 & 99.0 & 24.254 & 22.429 & 20.959 & 20.473 & 0.135 & 0.386 & 99.0 & 0.044 & 0.007 & 0.003 & 0.002 \\
\hline ......... & ........ & ........ & $\ldots \ldots$. & $\ldots \ldots$. & $\ldots \ldots$. & ........ & $\ldots \ldots$. & $\ldots \ldots$. & ........ & $\ldots \ldots$. & $\ldots \ldots$. & ........ & $\ldots \ldots$. & ....... \\
\hline
\end{tabular}

Magnitudes are in the $\mathrm{AB}$ photometric system. Negative magnitudes indicate upper limits, while mag $=99.0$ and err $=99.0$ indicate that the measure is not available.

Table 7. Extract of the GOODS-MUSIC web catalog: infrared photometry.

\begin{tabular}{|c|c|c|c|c|c|c|c|c|c|c|c|c|c|c|}
\hline ID & $\begin{array}{c}J \\
\mathrm{mag}\end{array}$ & $\begin{array}{c}H \\
\mathrm{mag} \\
\end{array}$ & $\begin{array}{c}K \mathrm{~s} \\
\mathrm{mag}\end{array}$ & $\begin{array}{l}3.6 \mu \\
\mathrm{mag}\end{array}$ & $\begin{array}{l}4.5 \mu \\
\mathrm{mag}\end{array}$ & $\begin{array}{l}5.8 \mu \\
\mathrm{mag}\end{array}$ & $\begin{array}{l}8.0 \mu \\
\mathrm{mag}\end{array}$ & $\begin{array}{c}J \\
\text { err }\end{array}$ & $\begin{array}{l}H \\
\text { err }\end{array}$ & $\begin{array}{l}K \mathrm{~s} \\
\text { err }\end{array}$ & $\begin{array}{c}3.6 \mu \\
\text { err }\end{array}$ & $\begin{array}{c}4.5 \mu \\
\text { err }\end{array}$ & $\begin{array}{c}5.8 \mu \\
\text { err }\end{array}$ & $\begin{array}{c}8.0 \mu \\
\text { err }\end{array}$ \\
\hline 10015 & 21.739 & 21.534 & 21.468 & 21.523 & 21.780 & 22.162 & 22.671 & 0.017 & 0.024 & 0.013 & 0.009 & 0.019 & 0.095 & 0.150 \\
\hline 10016 & 20.971 & 20.662 & 20.235 & 20.142 & 20.328 & 20.793 & 20.835 & 0.011 & 0.013 & 0.010 & 0.009 & 0.010 & 0.032 & 0.033 \\
\hline 10017 & 24.696 & 24.265 & 24.018 & 25.739 & -25.750 & -24.278 & -24.275 & 0.237 & 0.308 & 0.132 & 0.509 & 1.085 & 1.085 & 1.085 \\
\hline 10018 & 25.579 & -26.234 & 25.129 & 25.086 & 25.071 & 23.713 & -23.970 & 0.429 & 1.085 & 0.527 & 0.412 & 0.691 & 0.816 & 1.085 \\
\hline 10019 & -26.806 & 26.237 & 25.769 & 24.371 & 25.263 & 24.543 & 23.041 & 1.085 & 0.905 & 0.799 & 0.155 & 0.348 & 0.914 & 0.210 \\
\hline 10020 & 20.000 & 19.496 & 19.218 & 19.267 & 19.722 & 20.007 & 20.813 & 0.005 & 0.004 & 0.004 & 0.003 & 0.004 & 0.017 & 0.034 \\
\hline ......... & ........ & ........ & ........ & ........ & ........ & ....... & $\ldots \ldots$. & $\ldots \ldots$. & $\ldots \ldots$ & ........ & $\ldots \ldots$. & ........ & $\ldots \ldots$. & $\ldots \ldots$ \\
\hline
\end{tabular}

Magnitudes are in the AB photometric system. Negative magnitudes indicate upper limits, while mag $=99.0$ and err $=99.0$ indicate that the measure is not available.

which we developed to cope with the large dynamical and morphological range of the ACS mosaics. In addition to this, we identified the objects that are detected in the $K$ s images that escaped detection in the $z$ one. This double-pass procedure enabled us to obtain a unique catalog from which either a $z$-selected or a $K$ s-selected subsample can be easily extracted.

- A detailed set of simulations was executed to estimate the completeness limits of the samples. Although the effects of inhomogeneous coverage and depth mentioned above prevent a unique threshold from being adopted, we find that the typical completeness is at the level of $z \simeq 26$ or $K \mathrm{~s} \simeq 23.8$.

- The galaxy colours in the ACS images were estimated straightforwardly using the isophotal magnitudes provided by SExtractor. Conversely, the colour estimate on the IR or UV images, which have much poorer quality, was done using a specific "PSF-matching" software that we developed to this end and that we named ConvPhot. It is described at length in Sect. 4. As a result, all the 14847 galaxies (plus stars and AGNs) in the sample have a $U_{35}, U_{38}, B, V, i, z, J, K \mathrm{~s}, 3.6,4.5,5.8,8.0$ coverage, 9651 also have $U_{\text {VIMOS }}$ and 8441 have $H$.

- We cross-correlated our catalog with all the spectroscopic surveys available to date, assigning a spectroscopic redshift to more than 1000 sources in our catalog.

- The final catalog is made up of 14847 objects, with at least 72 known stars, 68 AGNs, and 928 galaxies with spectroscopic redshift (668 galaxies with reliable redshift determination).

- We applied our photometric redshift code to the 14 bands catalog. We applied a standard $\chi^{2}$ technique, choosing a set of synthetic templates drawn from the PEGASE2.0 synthesis model. The comparison with the spectroscopic sample shows that the quality of the resulting photometric redshifts is excellent, with an rms scatter of only 0.06 for the redshift interval $0<z<6$.

- The full multicolour GOODS-MUSIC catalog, including the redshift information (both spectroscopic and photometric) is made publicly available together with the software specifically designed for this purpose at the site http://lbc.oa-roma.inaf.it/mwcwi or at $\mathrm{CDS}^{4}$. An example of the public data can be found in Tables 5-7.

Acknowledgements. It's a pleasure to thank the GOODS Team for providing all the imaging material available worldwide. Observations were carried out using the Very Large Telescope at the ESO Paranal Observatory under Programme IDs LP168.A-0485 and ID 170.A-0788. We are grateful to the referee for an insightful report.

\section{References}

Arnouts, S., Vandame, B., Benoist, C., et al. 2001, A\&A, 379, 740 Beckwith, S. V. W., Caldwell, J., Clampin, M., et al. 2003, A\&AS, 202, 1705

${ }^{4}$ http://cdsweb.u-strasbg.fr/cgi-bin/qcat?J/A+A/449/951 
Bertin, E., \& Arnouts, S. 1996, A\&AS, 117, 393

Bruzual, G., \& Charlot, S. 2003, MNRAS, 344, 1000

Casertano, S., de Mello, D., Dickinson, M., et al. 2000, AJ, 120, 2747

Cimatti, A., Daddi, E., Mignoli, M., et al. 2002, A\&A, 381, L68

Cowie, L. L., Barger, A. J., Hu, E. M., Capak, P., \& Songaila, A. 2004, AJ, 127, 3137

Csabai, I., Budavari, T., Connolly, A. J., et al. 2003, AJ, 125, 580

Daddi, E., Cimatti, A., Renzini, A., et al. 2004, ApJ, 617, 746

Dale, D. A., Bendo, G. J., Engelbracht, C. W., et al. 2005, A\&AS, 206, 1004

Dickinson, M., \& the GOODS Legacy Team 2001, A\&AS, 198, 2501

Fazio, G. G., Hora, J. L., Allen, L. E., et al. 2004, ApJS, 154, 10

Ferguson, H. C., Dickinson, M., \& Williams, R. 2000, ARA\&A, 38, 667

Fernández-Soto, A., Lanzetta, K. M., \& Yahil, A. 1999, ApJ, 513, 34

Fioc, M., \& Rocca-Volmerange, B. 1997, A\&A, 326, 950

Fontana, A., D’Odorico, S., Poli, F., et al. 2000, AJ, 120, 2206

Fontana, A., Donnarumma, I., Vanzella, E., et al. 2003, ApJ, 594, L9

Fontana, A., Pozzetti, L., Donnarumma, I., et al. 2004, A\&A, 424, 23

Giacconi, R., Rosati, P., Tozzi, P., et al. 2000, A\&AS, 197, 9001

Giallongo, E., D’Odorico, S., Fontana, A., et al. 1998, AJ, 115, 2169

Giavalisco, M., \& the GOODS Team 2004, ApJ, 600, L93

Labbé, I., Franx, M., Rudnick, G., et al. 2003, AJ, 125, 1107

Lanzetta, K. M., Chen, H. W., Fernández-Soto, A., et al. 1999, ASPC, 193,544
Le Borgne, D., \& Rocca-Volmerange, 2002, A\&A, 386, 446

Le Fevre, O., Vettolani, G., Paltani, S., et al. 2004, A\&A, 428, 1043

Lu, N., Helou, G., Werner, M. W., et al. 2003, ApJ, 588, 199 Madau, P. 1995, ApJ, 441, 18

Madau, P., Pozzetti, L., \& Dickinson, M. 1998, ApJ, 498, 106

Mignoli, M., Cimatti, A., Zamorani, G., et al. 2005, A\&A, 437, 883

Mobasher, B., Idzi, R., Benítez, N., et al. 2004, ApJ, 600, L167

Papovich, C., Dickinson, M., \& Ferguson, H. C. 2001, ApJ, 559, 620

Phillipps, S., Driver, S. P., Couch, W. J., et al. 2000, MNRAS, 319, 807

Rana, N. C., \& Basu, S. 1992, A\&A, 265, 499

Steidel, C. C., Pettini, M., \& Hamilton, D. 1995, AJ, 110, 2519

Steidel, C. C., Adelberger, K. L., Giavalisco, M., Dickinson, M., \& Pettini, M. 1999, ApJ, 519, 1

Szokoly, G. P., Bergeron, J., Hasinger, G., et al. 2004, ApJS, 155, 271

Valdes, F. G. 2002, ADAAS 309, ed. Ranjan Gupta, Harinder P. Singh, \& Coryn A.L. Bailer-Jones, New Delhi, London

Vandame, B. 2002, SPIE, 4847, 123

Vanzella, E., Cristiani, S., Saracco, P., et al. 2001, AJ, 122, 2190

Vanzella, E., Cristiani, S., Fontana, A., et al. 2004, A\&A, 423, 761

Vanzella, E., Cristiani, S., Dickinson, M., et al. 2005, A\&A, 434, 53

Wolf, C., Meisenheimer, K., Roser, H. J., et al. 2001, A\&A, 365, 681 
A. Grazian et al.: GOODS-MUSIC multicolour catalog, Online Material p 1

\section{Online Material}




\section{Appendix A: True rms estimation with correlation matrix}

We provide detailed calculations here concerning the correlation of the noise and the theoretical interpretation.

Suppose we have a 1-dimensional distribution $x_{i}$, with $N$ random values. This distribution has a mean $m$ and an $\operatorname{rms} \sigma_{x}$. We define the correlation between adjacent pixels of distance equal to $j$ as:

$C_{j}=\frac{1}{N} \sum_{i=1}^{N}\left(x_{i}-m\right)\left(x_{i+j}-m\right)$.

The term $C_{0}$ is the variance of the distribution $\sigma_{x}^{2}$. If the pixels are all randomly distributed and uncorrelated, the general term $C_{j}$ behaves as a Delta function:

$C_{j}=\delta_{j} \sigma_{x}^{2}= \begin{cases}\sigma_{x}^{2} & \text { if } j=0 \\ 0 & \text { if } j \neq 0 .\end{cases}$

The terms $C_{j}$ should be nearly symmetric, if the number $N$ is large, $C_{-n} \sim C_{+n}$.

Considering the case of a correlated distribution $y$ built from this uncorrelated distribution, we have:

$y_{i}=\frac{\sum_{h=-k}^{+k} w_{h} x_{i+h}}{\sum_{h=-k}^{+k} w_{h}}$.

If the kernel $w$ conserves the flux, then $\sum_{h=-k}^{+k} w_{h}=1$ and $y_{i}=$ $\sum_{h=-k}^{+k} w_{h} x_{i+h}$.

We do not demonstrate (trivial) that the two distribution $x_{i}$ and $y_{i}$ have the same mean $m_{x}=m_{y}$. We consider here that the mean is null, $m=0$.

We now want to also evaluate the correlation coefficient $C_{j}$ for the new distribution $y_{i}$ :

$C_{j}^{y}=\frac{1}{N} \sum_{i=1}^{N} y_{i} y_{i+j}$,

which becomes

$\frac{1}{N} \sum_{i=1}^{N}\left[\sum_{h=-k}^{+k} w_{h} x_{i+h} y_{i+j}\right]=\sum_{h=-k}^{+k} w_{h} \frac{1}{N} \sum_{i=1}^{N} x_{i+h} y_{i+j}$

Now we can define

$U_{h j}=\frac{1}{N} \sum_{i=1}^{N} x_{i+h} y_{i+j}$,

which yields

$$
\begin{aligned}
& U_{h j}=\frac{1}{N} \sum_{i=1}^{N} x_{i+h}\left[\sum_{l=-k}^{+k} w_{l} x_{i+j+l}\right], \\
& U_{h j}=\sum_{l=-k}^{+k} w_{l}\left[\frac{1}{N} \sum_{i=1}^{N} x_{i+h} x_{i+j+l}\right],
\end{aligned}
$$

Table A.1. Correlation matrix of noise in the ACS $z$ band.

\begin{tabular}{cccc}
\hline \hline Column & -1 & 0 & +1 \\
\hline+1 & 0.06411 & 0.28940 & 0.06131 \\
0 & 0.28436 & 1.00000 & 0.28436 \\
-1 & 0.06131 & 0.28940 & 0.06411 \\
\hline
\end{tabular}

or

$U_{h j}=\sum_{l=-k}^{+k} w_{l} C_{j+l-h}^{x}$

Now we can sum over the terms $C_{j}^{y}$ :

$\sum_{j=-m}^{+m} C_{j}^{y}=\sum_{j=-m}^{+m}\left[\sum_{h=-k}^{+k} w_{h} \sum_{l=-k}^{+k} w_{l} C_{j+l-h}^{x}\right]$,

$\sum_{j=-m}^{+m} C_{j}^{y}=\sum_{h=-k}^{+k} w_{h} \sum_{l=-k}^{+k} w_{l} \sum_{j=-m}^{+m} C_{j+l-h}^{x}$.

But $\sum_{j=-m}^{+m} C_{j+l-h}^{x}=\sigma_{x}^{2}$; in that case we have

$\sum_{j=-m}^{+m} C_{j}^{y}=\sum_{h=-k}^{+k} w_{h} \sum_{l=-k}^{+k} w_{l} \sigma_{x}^{2}=\sigma_{x}^{2} \sum_{h=-k}^{+k} w_{h} \sum_{l=-k}^{+k} w_{l}$.

Using the fact that the kernel conserves the flux, $\sum_{h=-k}^{+k} w_{h}=1$ and $\sum_{l=-k}^{+k} w_{l}=1$, it finally yields

$\sum_{j=-m}^{+m} C_{j}^{y}=\sum_{j=-m}^{+m} C_{j}^{x}=\sigma_{x}^{2}$

Hence, we have demonstrated that the sum of the correlation coefficient $C_{j}$ gives the total variance of a distribution, whether correlated or not. Indeed, since $C_{0}^{y}=\sigma_{y}^{2}$ and $C_{0}^{y} \leq \sum_{j=-m}^{+m} C_{j}^{y}$, we can conclude that the variance of a correlated distribution is less than the variance of the original distribution, or true variance, and it is an underestimated value for the rms of the data.

These results are derived for a discrete distribution, but they can be generalised to the continuum case with the help of integrals and Fourier Transform. All these results derived from a 1-dimensional distribution can be easily generalised for a 2-dimensional distribution, which in our case is a reduced image, where the noise between adjacent pixels is correlated, due to the algorithms used to sum the dithered exposures and the seeing.

In the $z$ band, the resulting kernel (Table A.1) is positive in the central $3 \times 3$ pixels, and fades rapidly to zero at large distances. If one only considers the central $3 \times 3$ pixels and normalises the peak value to 1 , it results a correlation matrix as in Table A.1.

The sum of all the terms gives the ratio between the true and the measured variance $\left(\sigma_{x}^{2} / \sigma_{y}^{2}\right)$ of the image. 